2018-10-15

Uncertainty and Sensitivity Analysis of

Energy Assessment for Office Buildings

based on Dempster-Shafer Theory

Tian, W

http://hdl.handle.net/10026.1/12198

10.1016/j.enconman.2018.08.086

Energy Conversion and Management

Elsevier

All content in PEARL is protected by copyright law. Author manuscripts are made available in accordance with publisher policies. Please cite only the published version using the details provided on the item record or document. In the absence of an open licence (e.g. Creative Commons), permissions for further reuse of content should be sought from the publisher or author. 


\title{
Uncertainty and Sensitivity Analysis of Energy Assessment for Office Buildings based on Dempster-Shafer Theory
}

\author{
Wei $\operatorname{Tian}^{1,2}$ *, Pieter de Wilde ${ }^{3}$, Zhanyong Li $^{1,2}$, Jitian Song ${ }^{1,2}$, Baoquan Yin ${ }^{4}$ \\ ${ }^{1}$ Tianjin Key Laboratory of Integrated Design and On-line Monitoring for Light Industry \& Food \\ Machinery and Equipment, College of Mechanical Engineering, Tianjin University of Science and \\ Technology, Tianjin 300222, China \\ ${ }^{2}$ Tianjin International Joint Research and Development Center of Low-Carbon Green Process Equip- \\ ment, Tianjin 300222, China \\ ${ }^{3}$ Chair of Building Performance Analysis, Environmental Building Group, University of Plymouth, \\ Plymouth, Devon PL4 8AA, United Kingdom \\ ${ }^{4}$ Tianjin Architecture Design Institute, 300074, Tianjin, China
}

\begin{abstract}
Uncertainty and sensitivity analysis of building energy has become an active research area in order to consider variations of input variables and identify key variables influencing building energy. When there is only limited information available for uncertainty of building inputs, a specific probability for a given variable cannot be defined. Then, it is necessary to develop alternative approaches to probabilistic uncertainty and sensitivity analysis for building energy. Therefore, this paper explores the application of the Dempster-Shafer theory (DST) of evidence to conduct uncertainty and sensitivity analysis for buildings. The DST method is one of imprecise probability theories to allow combining uncertainty from different sources in terms of interval-valued probabilities in order to construct the belief and plausibility (two uncertainty measures) of system responses. The results indicate that the DST uncertainty analysis in combination with machine learning methods can provide fast and reliable information on uncertainty of building energy. It is recommended that at least two inherently different learning algorithms should be applied to provide robust simulation results of building energy. A spectrum of distributions should be implemented in global sensitivity analysis with the DST method because there are no specific distributions for intervals of input factors. Moreover, the stability of results from uncertainty and sensitivity analysis should be assessed when applying the DST method in building energy analysis.
\end{abstract}

Keywords: Uncertainty analysis; building performance; Dempster-Shafer theory; sensitivity analysis; machine learning

*Corresponding author: Wei Tian, Tel: +86 (022) 60600705, Fax: +86 (022) 60600705

Email: tjtianjin@gmail.com 


\begin{tabular}{|c|c|}
\hline \multicolumn{2}{|c|}{ Abbreviations } \\
\hline BPA & Basic probability assignment \\
\hline $\mathrm{CBF}$ & Cumulative belief function \\
\hline $\mathrm{CCBF}$ & Complementary cumulative belief function \\
\hline $\mathrm{CCPF}$ & Complementary cumulative plausibility function \\
\hline $\mathrm{CDF}$ & Cumulative density function \\
\hline $\mathrm{CHP}$ & Combined heat and power \\
\hline $\mathrm{CL}$ & Cooling set-point temperature $\left({ }^{\circ} \mathrm{C}\right)$ \\
\hline $\mathrm{CPF}$ & Cumulative plausibility function \\
\hline CSWD & Chinese standard weather data \\
\hline DST & Dempster-Shafer theory \\
\hline ED & Equipment peak value $\left(\mathrm{W} / \mathrm{m}^{2}\right)$ \\
\hline FT & Infiltration rate $(\mathrm{ACH})$ \\
\hline HT & Heating set-point temperature $\left({ }^{\circ} \mathrm{C}\right)$ \\
\hline $\mathrm{LD}$ & Lighting power density $\left(\mathrm{W} / \mathrm{m}^{2}\right)$ \\
\hline MARS & Multivariate adaptive regression splines \\
\hline OD & Occupancy density (people/m²) \\
\hline RMSE & Root mean square error \\
\hline SHGC & Solar heat gain coefficient \\
\hline SVM & Support vector machine \\
\hline VAV & Variable air volume system \\
\hline
\end{tabular}




\section{Introduction}

Building energy is affected by a number of inherently uncertain variables, including weather conditions, internal heat gains, and occupant behaviours [1,2]. Therefore, uncertainty analysis of building energy has become an active research field [3-5]. Most previous studies have implemented probabilistic uncertainty methods to consider the influences of these uncertain parameters [6-8]. Urbanucci and Testi [9] use the Monte Carlo risk analysis to estimate the long-term uncertainty of energy demands for a hospital facility in order to optimize the size of CHP (combined heat and power) system. Tian et al. [10] consider the influences of variations of building form on energy performance of buildings located at Harbin (China) based on the Monte Carlo sampling method. Faggianelli et al. [11] implement sampling-based sensitivity analysis by regarding input factors as uniform or normal distributions. Hopfe and Hensen [12] assume the normal distributions for input factors to assess energy performance of an office building using the Latin hypercube sampling method. These examples demonstrate that probabilistic uncertainty and sensitivity analyses have become very popular and widely used in the field of building performance simulation. However, variations of building variables are difficult to obtain and it can be a challenge to gather sufficient information for the definition of a specific probability (such as uniform, normal, triangle, and lognormal) when predicting energy use, especially in the stage of building design [13]. Hence, the alternative approach to probabilistic analysis is needed to handle the imprecise building data in properly estimating energy performance of buildings.

The Dempster-Shafer theory (DST) of evidence can be regarded as a generalization of classical probability theory that allows one to deal with the imprecise information on data, often in the form of interval-valued data. The mathematical foundations of DST analysis have been well established [14] and the DST approach has been used in various fields, including studies on reliability of pressure vessels [15], petroleum engineering [16], urban environment [17], and computer voice detection [18]. More recently, the DST analysis is also being applied to the analysis of building energy. Tian et al. [3] implement the DST to assess uncertainty of energy performance for an office building using the EnergyPlus program. Four scenarios are used in their research to represent the level of availability for uncertain inputs from the simple to detailed information. Chaney et al. [19] use the DST to add multiplesensor data in a house simulation model. They found that the evidence theory is a reasonable approach for providing rich information about occupant interaction with systems in the house. Kim et al. [20] report that the DST can be used to effectively combine uncertainties from five experts into single uncertainty when predicting energy use for a 33-storey office building in Seoul, Korea.

These previous studies provide valuable information on the implementation of DST analysis in building energy assessment. However, there are several issues that have not been explored when applying the DST method in building energy assessment. One issue is how to reduce high computational cost of DST analysis in building simulation using engineeringbased energy models. A large number of simulation runs are usually required to provide the minimum and maximum output values in order to obtain the stable results of output range for the DST method. Another issue is how to implement sensitivity analysis within the context of DST analysis in assessing building energy performance. The sampling-based sensitivity analysis requires the structured distributions of input variables to obtain a matrix of inputs and outputs. The DST method, however, does not include specific distributions for the data within the intervals.

Therefore, this paper explores a systematic approach towards implementation of the DST method in uncertainty and sensitivity analysis of building energy when only limited information on building input variables is available. An office building located in Tianjin (China) is used as a case study to demonstrate the suitability of DST method in assessing 
building energy performance. The building energy simulation is carried out with the EnergyPlus program [21]. The originality of this paper is two-fold: (1) implementation of global sensitivity analysis in conjunction with the DST analysis in assessing building energy performance; (2) demonstration of using machine learning models to reduce high computational cost of building energy simulation for both uncertainty and sensitivity analysis within the DST analysis. Moreover, this research discusses two important issues in the application of DST analysis: how to choose reliable machine learning models and how to assess the stability of uncertainty and sensitivity analysis. This provides practical guidance in applying the DST method into building energy assessment. The combination of DST and machine learning algorithm can significantly expedite computation, which can make DST analysis feasible in building energy assessment. However, a number of machine learning models should be evaluated to choose suitable ones for replacing building energy models on a case-by-case basis. More discussion on the method used will be presented in section 2 .

The remaining parts of this paper are structured as follows. Section 2 describes the statistical methods applied in this research, including the DST analysis, machine learning models, and sensitivity analysis. Section 3 presents a case study of building energy model to implement DST analysis. Section 4 discusses the results from these three types of statistical approaches when assessing the energy performance of an office building. Section 5 presents the conclusions and further research required in this field.

2 Method

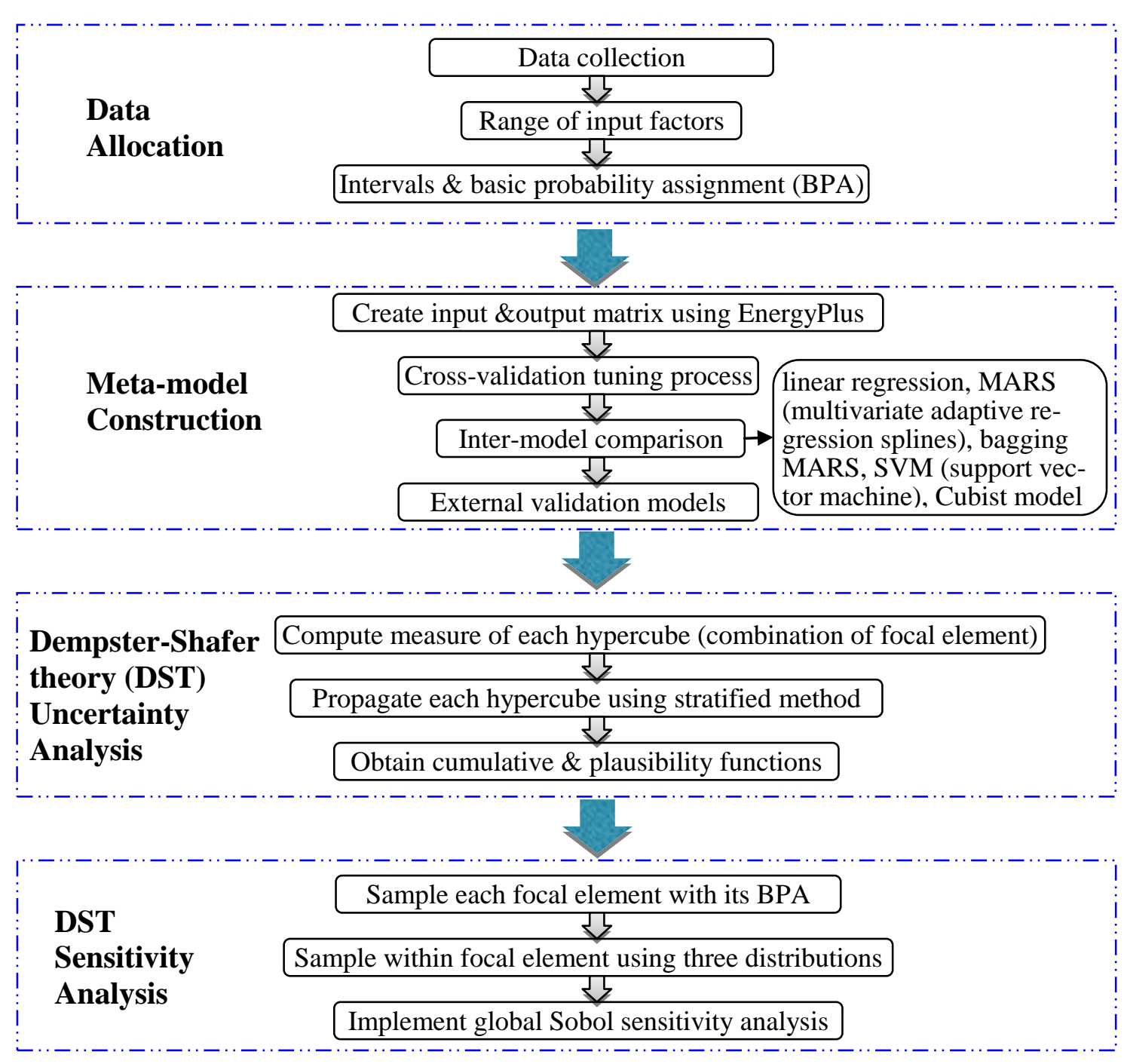


The computational procedure used in this study is shown in Figure 1. The first step is to collect the data for uncertainty and sensitivity analysis of building energy assessment. The data should be processed based on the requirement of the DST analysis (as described in sections 3). The second step is to create fast-computing machine learning models based on the engineering-based energy models (sections 2.2). These computationally cheap models then will be used for the uncertainty and sensitivity analysis of DST method. The third step is to implement DST uncertainty analysis with the data obtained from the first step using the learning models from the second step (section 2.1). The fourth step is to apply global sensitivity analysis in conjunction with the DST analysis based on the machine learning models obtained from the second step (section 2.3).

123

124

125

126

127

128

129

130

131

132

133

134

135

136

137

138

139

140

141

142

143

144

145

146

\subsection{Dempster-Shafer Theory}

For most engineering problems, the relationships among inputs and outputs can be written as,

$$
y=f(x)
$$

where $x$ is a vector of system inputs, $y$ is a vector of system outputs, and $f$ is a function to describe the relationships among $x$ and $y$. In this case study, $x$ represents input variables in building energy assessment, such as occupant density, equipment heat gains, and heating setpoint temperature, while $y$ is energy performance, such as annual heating energy, annual cooling energy, annual total electricity, or carbon emissions. The $f$ functions in this research are to represent complex relationships among building input variables and energy performance that can be computed using building simulation programs, such as EnergyPlus, ESP-r, and DOE-2 [22].

It is common practice to define probabilities for building input variables in uncertainty analysis of building performance. The sampling-based probabilistic uncertainty propagation could be used to obtain uncertain performance of buildings by running building energy models with a large number of times. However, in the case of limited information for these input data, it may be difficult to justify the choices of specific probabilities. As a result, a less structured representation of uncertainty for building input factors is needed instead of specific probabilities. The Dempster-Shafer theory (DST) of evidence is a generalization of classical probability theory to handle the imprecise information on input data [14].

For the DST analysis, an evidence space for input data $x$ is specified as a triple $\left(\mathrm{X}, \mathrm{X}_{\mathrm{E}}\right.$, $\mathrm{m}_{\mathrm{EX}}$ ), where $\mathrm{X}$ is the set of all possible values (i.e sample space), $\mathrm{X}_{\mathrm{E}}$ is a set of subsets of $\mathrm{X}$ (i.e. focal elements), and $m_{E X}$ is a function for a subset $U$ of $X$ (i.e. basic probability assignment, BPA).

$$
\begin{aligned}
& \mathrm{m}_{\mathrm{EX}}(\mathrm{U})>0 \text { if } \mathrm{U} \subset \mathrm{X} \text { and } \mathrm{U} \in \mathrm{X}_{\mathrm{E}} \\
& \mathrm{m}_{\mathrm{EX}}(\mathrm{U})=0 \text { if } \mathrm{U} \subset \mathrm{X} \text { and } \mathrm{U} \notin \mathrm{X}_{\mathrm{E}}
\end{aligned}
$$

The $\mathrm{m}_{\mathrm{EX}}(\mathrm{U})$ function denotes the amount of information assigned to U. Similar to the probability theory, the sum of basic probability assignment equals one. However, in the DST method, there are two uncertainty measures: belief and plausibility defined as follows,

$$
\begin{aligned}
& \operatorname{Bel}_{X}(\mathrm{U})=\sum_{V \subset \mathrm{U}} m_{E X}(V) \\
& \mathrm{Pl}_{X}(\mathrm{U})=\sum_{V \cap \mathrm{U} \neq \varnothing} m_{E X}(V)
\end{aligned}
$$

$\operatorname{Bel}_{X}(U)$ can be interpreted as a measure of the amount of information to support $\mathrm{U}$ containing true values, while $\operatorname{Plx}(U)$ represents the absence of information to support $\mathrm{U}$ containing false values. In a similar way, the uncertainty of system output defined in Eq.(1) can be obtained with an evidence space of triple $\left(\mathrm{Y}, \mathrm{Y}_{\mathrm{E}}, \mathrm{m}_{\mathrm{EY}}\right)$. The resultant uncertainty of outputs can be summarized with a cumulative belief function (CBF) and a cumulative 
plausibility function (CPF) by the corresponding beliefs and plausibilities. More detailed

As shown in Figure 1, the computation procedure for the DST uncertainty analysis can be divided into three steps. The first step is to define a hypercube that is the combination of focal elements for input variables and then calculate the composite evidential measure (i.e. BPA) of each hypercube. For the case study building as will described in section 3, the numbers of hypercubes in case A and case B are 32 and 512, respectively. These two values can be calculated based on the fundamental of combination from the numbers of intervals listed in Table 1 and Table 2.

The second step is to compute the minimum and maximum values of the system response in each hypercube, which are the most computationally intensive procedure in the DST analysis. The computational methods available are optimization, sampling, and vertex techniques $[14,24]$. To reduce computation cost, machine learning algorithms are used to provide reliable results with fast computing as will be described in section 2.2. The sampling method is chosen in this paper since the whole output space can be sufficiently explored by using machine learning energy models. The conventional probability sampling method [25, 26] is used to obtain the uncertainty of outputs for each hypercube cell. The uniform distribution for all input variables is assumed to propagate input uncertainty to output uncertainty and then find the minimum and maximum output values for this hypercube space. Note that this does not mean the distribution within intervals is uniform and the purpose of assuming uniform distribution is only to obtain the minimum and maximum values within the intervals. Latin Hypercube sampling with a sampling size of 10,000 is used due to its high stratification. Discussion of convergence of outputs with sample size will be presented in section 4.2.1.

The third step is to form the cumulative belief (CBF) and plausibility functions (CPF) by aggregating the minimum and maximum values of system response obtained from the second step. The uncertainty results with the DST method are bounded between the CBF and CPF. The CBF is a lower bound on a probability value consistent with the evidence space, whereas the CPF is an upper bound on a probability value consistent with the evidence space. Hence, uncertainty results can be interpreted by the CBF (the smallest probability) and CPF (the largest probability) that are combined together to have the complete information of all possible output values (i.e. energy use in this case study). Similar to the probability theory, the CCBF (complementary cumulative belief function) and CCPF (complementary cumulative plausibility function) may be more useful in the field of risk analysis or reliability analysis.

\subsection{Machine learning algorithms}

Machine learning is used to create reliable and fast-computing models (also called metamodels or surrogate models) based on the inputs and outputs computed from the EnergyPlus program. In this study, 400 EnergyPlus models are used to construct a matrix containing inputs variables and outputs for this office building using the Sobol sequence. This Sobol sequence is a quasi-random low-discrepancy sequence with a better performance in comparison with the Monte Carlo sampling [27, 28]. The 400 simulation runs of EnergyPlus models are used in this case study based on preliminary studies to create reliable meta-models of building energy use. This simulation number is higher than ten times of input variables used in most of building energy simulation studies [13]. The simulation number of energy models required for creating accurate energy models can be evaluated using the RMSE (root mean square error) of energy models. In this study, the RMSE for three performance indicator has become stable after around 300 times and the extra 100 times of simulation models (total number 400) are used to ascertain better performance of energy analysis. The determination of simulation number for constructing reliable meta-models is likely to be problem dependent, depending on number of input variables, simulation output, complexity among inputs and 
outputs, and accuracy required by building projects. The $\mathrm{R}$ caret package [29] is used to create these machine learning models in this study. The R caret package combines more than

Five machine learning algorithms have been selected since they have better performance in terms of predictive capability and are also widely used in the field of building energy analysis $[30,31]$. These five models are linear regression, MARS (multivariate adaptive regression splines), bagging MARS, SVM (support vector machine), and Cubist model. The reason for exploring these five options is as follows. (1) The linear model is still used here because the linear model has good performance with better interpretation [32]. (2) MARS creates a piecewise linear model to replace original predictors with new surrogate features to account for non-linear effects [33]. If necessary, the interaction terms of these new features can be also considered in MARS models to further improve predictive performance. The number of new features and the number of degrees of interactions can be determined using an automatic pruning procedure. (3) The bagging MARS approach implements the bagging (bootstrap aggregating) technique to stabilize the predictive results from MARS models. The bagging technique, an ensemble learning method, simply creates a number of new data set using the bootstrap method (i.e. randomly sampling with replacement) to crease a number of corresponding models instead of only one regression model [33]. Then the prediction results are averaged from these regression models to reduce the variance of outcomes. A disadvantage of the bagging technique is high computational cost because more computational time is required with an increase in the number of bootstrap samples. A multicore workstation is used here to expedite the calculation using parallel computing. (4) The SVM is similar to robust regression that tries to mitigate the influence of influential observations. Several kernel functions (polynomial, radial basis, hyperbolic tangent) are available in SVM to encompass nonlinear functions of inputs. The radial basis function is chosen in this study based on the suggestion from Kuhn and Johnson [33]. (5) The Cubist belongs to the rule-based models with the boosting technique. The boosting technique is one of ensemble methods to provide the unequally weights for different models in terms of model errors [3]. More detailed information on these machine leaning techniques is available in [33, 34].

In the five models above, model variables need to be tuned except for the linear model. The cross-validation method is used to find the optimal values for these models. The cross validation in this study is based in randomly dividing the original data set into ten sets of roughly equal size (also named ten-fold) [34]. Then one new data set is used as a test set to assess the performance of regression models obtained from the remaining nine data sets as training data. This process repeats ten times until all the ten data sets are used as test sets. To further test the predictive performance of the optimal model for five algorithms, an extra 200 EnergyPlus models are simulated except for the 400 simulation that are used for regression. Two measures are used to assess predictive performance of regression models: RMSE (root mean square error) and $\mathrm{R}^{2}$ (coefficient of determination). RMSE is widely used in the field of machine learning and is the absolute fit measure how the regression model predicts the outcomes. The lower RMSE, the better regression model is. $\mathrm{R}^{2}$ is the relative measure to account for the proportion of total variance explained by the model. The higher $\mathrm{R}^{2}$ indicate $\mathrm{a}$ better regression model.

The choice of suitable machine learning models involves a lot of efforts, which is related to the prior knowledge of both building physics and machine learning algorithm. For instance, if there are interactions among input variables on building energy use, it is necessary to choose machine learning models that can consider interactions. For the MARS approach, second or higher degree terms should be added to tune optimal models. This is the case for London domestic gas use influenced by a number of factors, including dwelling type, household composition, and building area [30]. However, if there are no strong interactions, 
the MARS model without second degree terms should be used since more terms actually deteriorate model predictive performance. This is the case for assessing annual heating and cooling energy of an office building located in London [35]. When there exists highly nonlinear relationships between inputs and output in building energy performance, nonparametric machine learning models usually perform better than linear models. However, if there are approximately linear relationships in building energy analysis, linear models would have more robust performance in comparison with most of complex non-parametric relationships. This has been confirmed in [36] to assess energy performance of campus buildings at Georgia Institute of Technology, USA. If using support vector machine, linear kernel function should be used instead of non-linear polynomial or radial basis functions. Tian et al [36] also discuss another important issue on correlation of input variables, which usually leads to unstable meta-models. For instance, equipment heat gains are usually associated with lighting use in office buildings. Then, the principal component approach or partial least square method can be used to reduce the number of correlated variables to increase the stability of meta-models. More research is required to choose suitable machine learning algorithms based on building features, building type, and thermal performance of buildings,

\subsection{Sensitivity analysis for Dempster-Shafer Theory}

The DST method causes two issues in implementing sensitivity analysis in building energy assessment. The first issue is that the DST analysis does not assume any distribution for the intervals of input variables. However, the sampling-based global sensitivity analysis requires to have the specific distributions for a variable, which may have significant influence on sensitivity results [37]. The second issue is the high computational cost for sensitivity analysis due to the nature of DST analysis as discussed in section 2.1. For the first issue, the sensitivity method used here is based on the recommendation from Helton et al. [38] to specify a spectrum of distributions to represent possible variations within the intervals of focal elements. Three types of distributions are considered to cover the larger values, middle value, and lower values with the left quadratic, uniform, and right quadratic distributions, respectively. For the second issue, similar to the DST uncertainty analysis, reliable machine learning models as described in section 2.2 are used for running global sensitivity analysis instead of the engineering-based EnergyPlus models. The fast-computing machine learning models can assure the convergence of the global sensitivity analysis by running a large number of times of simulation models.

The computational procedure for sensitivity analysis is illustrated in Figure 1. The first step is to sample each focal element with its BPA using random sampling. The next step is to sample within the corresponding focal element using three types of distributions (left quadratic, uniform, and right quadratic), respectively. The reasons for choosing these three distributions are to cover as many as possible situations within intervals defined from the first step. The left quadratic distribution can emphasize the smaller values with each focal element, whereas the right quadratic distribution can emphasize the larger values with each focal element. The uniform distribution can cover the whole range of each focal element. The density functions for left, uniform, and right distributions, respectively, are

$$
\begin{aligned}
& f_{\text {left }}(x)=3(b-x) /(b-a)^{3} \\
& f_{\text {uniform }}(x)=1 /(b-a) \\
& f_{\text {right }}(x)=3(x-a) /(b-a)^{3}
\end{aligned}
$$

where $\mathrm{a}$ and $\mathrm{b}$ are minimum and maximum values, respectively, within an interval defined in the first step. More detailed descriptions on these distributions are available in [38].

The final step is to implement global Sobol sensitivity analysis with machine learning models to provide importance ranking of input variables [39]. The Sobol sensitivity method is 
one of variance-based approach to decompose the variance of output to the corresponding input variable. The detailed procedure for the Sobol sensitivity analysis is available in [37]. Two sensitivity indicators are often used in the variance-based method: main effect and total effect. The main effect represents the effects of one individual variable without considering other variables, whereas the total effect is due to the effects of this specific variable and interactions with the other variables. R sensitivity package [40] is used here to implement the Sobol sensitivity analysis.

Note that the sensitivity analysis used here is different from quantitative risk assessment in which the probability of input variables need to be specified in the first place. For the DST sensitivity analysis, there is no assumption on the probability for input variables. The results from quantitative risk assessment are similar to the probabilistic sensitivity analysis by considering only one specified probability. In contrast, the results from the DST sensitivity analysis are the combined ranking importance to consider all the possibilities within the intervals. Hence, the results from the DST sensitivity analysis depend on a number of factors, including the BPA and the relationships among inputs and outputs, but not on the specification of probabilities within these intervals of input variables.

\section{A case study of building energy model}

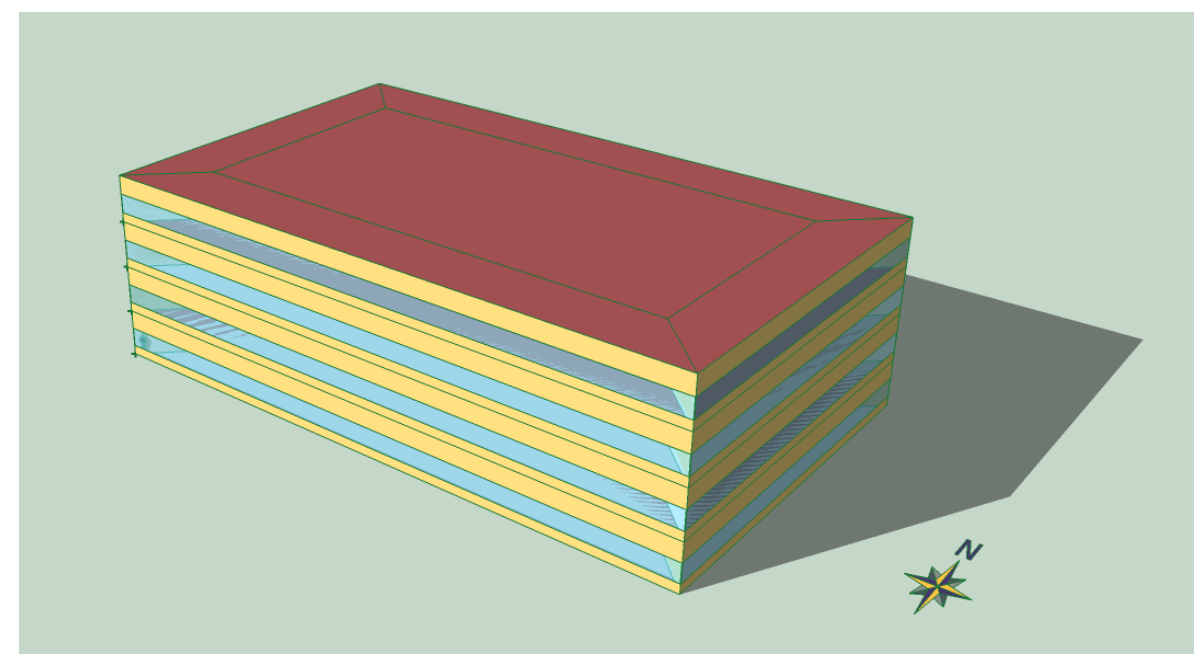

Figure 2. An office building used for this research

Figure 2 illustrates an office building studied in this paper. It is a four-storey building with a total floor area of $6,000 \mathrm{~m}^{2}$. The window-wall ratio is $40 \%$. The thermal properties of building envelope are commensurate with the requirements of energy efficiency for office buildings in China [41]. The U-values for wall and roof are taken as 0.45 and $0.23 \mathrm{~W} / \mathrm{m}^{2} \mathrm{~K}$, respectively. The U-value and SHGC (solar heat gain coefficient) for windows are 2.40 $\mathrm{W} / \mathrm{m}^{2} \mathrm{~K}$ and 0.35 , respectively. A VAV (variable air volume) air system with perimeter hydronic baseboard heaters is used to provide ventilation, heating, and cooling to maintain indoor thermal comfort. A gas boiler is used to supply hot water, and a centrifugal chiller with air cooling is used to supply chilled water for the VAV system.

Internal heat gains for occupants, lighting, and equipment are derived from two Chinese standards [41, 42] and expert opinions as listed in Table 1. The interpretation of values in Table 1 will be discussed in the end of this subsection. The office building is located in Tianjin, China and the typical year weather data (CSWD, Chinese standard weather data) is obtained from the EnergyPlus website [21]. The climate in Tianjin has a cold, windy winter and hot, humid summer, which requires heating in winter and cooling in summer.

The EnergyPlus V8.8 program is used to simulate the thermal behaviours of the building [21]. EnergyPlus is widely used in the field of building energy analysis and has been validated extensively. Typical one-core and four-perimeter zones are used for zoning this 
building when creating an energy model. Three performance measures are annual heating energy, annual cooling energy, and annual carbon emissions normalized by the floor area. Heating and cooling energy values are directly obtained from the simulation results of EnergyPlus models. Carbon emissions are calculated by multiplying the carbon emission factors of electricity use $\left(1.00 \mathrm{kgCO}_{2} / \mathrm{kWh}\right)$ [43] and natural gas $\left(0.20 \mathrm{kgCO}_{2} / \mathrm{kWh}\right)$ [44], respectively, with annual electricity and gas use from the results of EnergyPlus models. Note that other performance measures can be also used for this method, such as overheating risk in natural ventilation buildings.

Table 1. Intervals and BPA (basic probability assignment) from Expert I and Expert II

\begin{tabular}{cccccc}
\hline \multirow{2}{*}{ Variable } & Short & \multicolumn{2}{c}{ Expert I (case A) } & \multicolumn{2}{c}{ Expert II } \\
\cline { 3 - 6 } & names & Intervals & $B P A$ & Intervals & $B P A$ \\
\hline $\begin{array}{c}\text { Infiltration rate } \\
(\mathrm{ACH})\end{array}$ & FT & {$[0.3,0.4],[0.4,0.5]$} & $0.6,0.4$ & {$[0.3,0.4]$} & 1 \\
$\begin{array}{c}\text { Equipment peak } \\
\text { value }\left(\mathrm{W} / \mathrm{m}^{2}\right)\end{array}$ & ED & {$[13,15],[16,17]$} & $0.6,0.4$ & {$[14,15],[15,16]$} & $0.8,0.2$ \\
$\begin{array}{c}\text { Lighting power } \\
\text { density }\left(\mathrm{W} / \mathrm{m}^{2}\right)\end{array}$ & LD & {$[6,8],[7,9]$} & $0.3,0.7$ & {$[6,7],[7,8]$} & $0.4,0.6$ \\
$\begin{array}{c}\text { Occupancy densi- } \\
\left.\text { ty (people/m }{ }^{2}\right)\end{array}$ & OD & {$[8,10],[11,12]$} & $0.5,0.5$ & {$[9,10],[10,11]$} & $0.8,0.2$ \\
$\begin{array}{c}\text { Heating set-point } \\
\text { temperature }\left({ }^{\circ} \mathrm{C}\right)\end{array}$ & HT & {$[19,21]$} & & & 1 \\
$\begin{array}{c}\text { Cooling set-point } \\
\text { temperature }\left({ }^{\circ} \mathrm{C}\right)\end{array}$ & CL & {$[24,25],[25,26]$} & $0.6,0.4$ & {$[24,25]$} & 1 \\
\hline
\end{tabular}

Table 2. The combined intervals and BPA (basic probability assignment) for case B from two experts

\begin{tabular}{ccc}
\hline Variable & Intervals & BPA \\
\hline Infiltration rate $(\mathrm{ACH})$ & {$[0.3,0.4],[0.4,0.5]$} & $0.8,0.2$ \\
Equipment peak value $\left(\mathrm{W} / \mathrm{m}^{2}\right)$ & {$[13,15],[14,15],[15,16],[16,17]$} & $0.3,0.4,0.1,0.2$ \\
Lighting power density $\left(\mathrm{W} / \mathrm{m}^{2}\right)$ & {$[6,7],[6,8],[7,8],[7,9]$} & $0.2,0.15,0.3,0.35$ \\
Occupancy density (people $\left./ \mathrm{m}^{2}\right)$ & {$[8,10],[9,10],[10,11],[11,12]$} & $0.25,0.4,0.1,0.25$ \\
Heating set-point temperature $\left({ }^{\circ} \mathrm{C}\right)$ & {$[19,21],[20,21]$} & $0.5,0.5$ \\
Cooling set-point temperature $\left({ }^{\circ} \mathrm{C}\right)$ & {$[24,25],[25,26]$} & $0.8,0.2$ \\
\hline
\end{tabular}


The uncertain input factors considered in this study are listed in Table 1. The purpose of the case study is to explore how the DST can help to provide more reliable simulation outputs by considering uncertainty of new buildings in the preliminary design stage. These variables are closely related to occupant behaviour, including infiltration rate, equipment heat gains, lighting heat gains, heating \& cooling set-point temperatures. The information on these input variables is obtained from two experts in the area of building energy engineering, Expert I and Expert II as summarized in Table 1. For the infiltration rate, Expert I states that the actual infiltration rate is in one of two contiguous intervals: in the interval $[0.3,0.4]$ with a $60 \%$ level of subjective belief (named as basic probability assignment in evidence theory), or in the interval $[0.4,0.5]$ with a $40 \%$ level of subjective belief. In contrast, in Expert II's opinion, the infiltration rate lies in the interval $[0.3,0.4]$, with a $100 \%$ level of subjective belief. The other values in Table 1 can be interpreted in the same way. Based on the suggestion from these two experts and previous studies [8, 13], the infiltration rate is treated as a constant ACH (air exchange per hour) value in a whole year in this research since the infiltration rate is very uncertain, depending on building age, construction quality, building use, and weather conditions [13].

Two cases (named as case A and case B) are considered in this paper to represent two different uncertain situations. Case A is directly obtained by the opinion of the Expert I as listed in Table 1. Case B is derived by combining the opinions from Expert I and Expert II as summarized in Table 2 in which the input variables can be explained in the same way as the values in Table 1. It is assumed that the two sources are weighted equally since both Expert I and Expert II are senior building engineers. The detailed calculation procedure is available in a book chapter written by Oberkampf and Helton [23]. A number of methods are available to combine the evidence from different sources; please refer to [45, 46].

In order to compare the results from DST and probability-based analysis, the simulation results from uniform distributions are used as a special case for probability-based analysis to represent the results for conventional probabilistic method. The corresponding uncertainty results are named as cumulative distribution function (CDF) in this research. Note that the results from the DST method are interval-based for a specific probability, whereas the results from the probabilistic method are specific values for a given probability.

\section{Results and discussion}

\subsection{Performance comparison of machine learning models}

Figure 3 shows the comparisons of predictive performance of five machine learning models (as described in section 2.2) for heating, cooling, and carbon emissions in order to choose reliable models for uncertainty and sensitivity analysis based on the Dempster-Shafer theory of evidence. The variations of RMSE (root mean square error) and $\mathrm{R}^{2}$ are expressed as 95\% confidence interval of two statistics in this study. A reliable machine learning model should have low RMSE values and high $\mathrm{R}^{2}$. Moreover, the variations for these two measures should be also low to provide stable estimation of simulation outputs for buildings in this case study.

Figure 3a indicates that the Cubist model performs the best amongst the five models for estimating heating energy. The mean value of corresponding RMSE for heating energy is only $0.14 \mathrm{kWh} / \mathrm{m}^{2}$ and the variation of RMSE is also small in terms of $95 \%$ confidence interval. The next two models are the MARS and bagging MARS models with similar accuracy, which indicates that the bagging technique does not significantly improve predictive accuracy in this case study. The linear and SVM models do not perform well for predicting annual heating energy use. To further validate regression models, the extra 200 simulation runs that are not used to obtain regression models are applied to the external validation of models. The statistics from this external validation are listed in Table 3 in which the Cubist model has the best performance in terms of both $\mathrm{R}^{2}$ and RMSE. The MARS and 
bagging MARS have similar predictive capability for heating energy. These conclusions are the same as those obtained in the internal cross-validation method, which indicates that the 409 Cubist and MARS regression can be used to provide reliable heating energy use for this office 410 building.
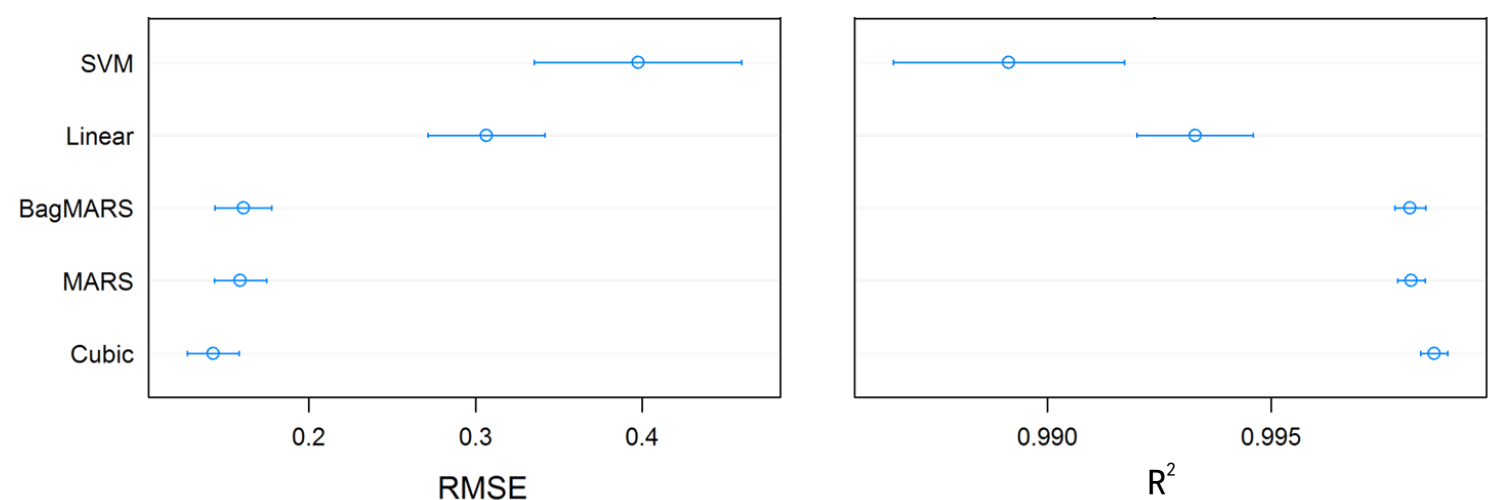

Confidence Level: $\mathbf{0 . 9 5}$

(a) Heating energy use
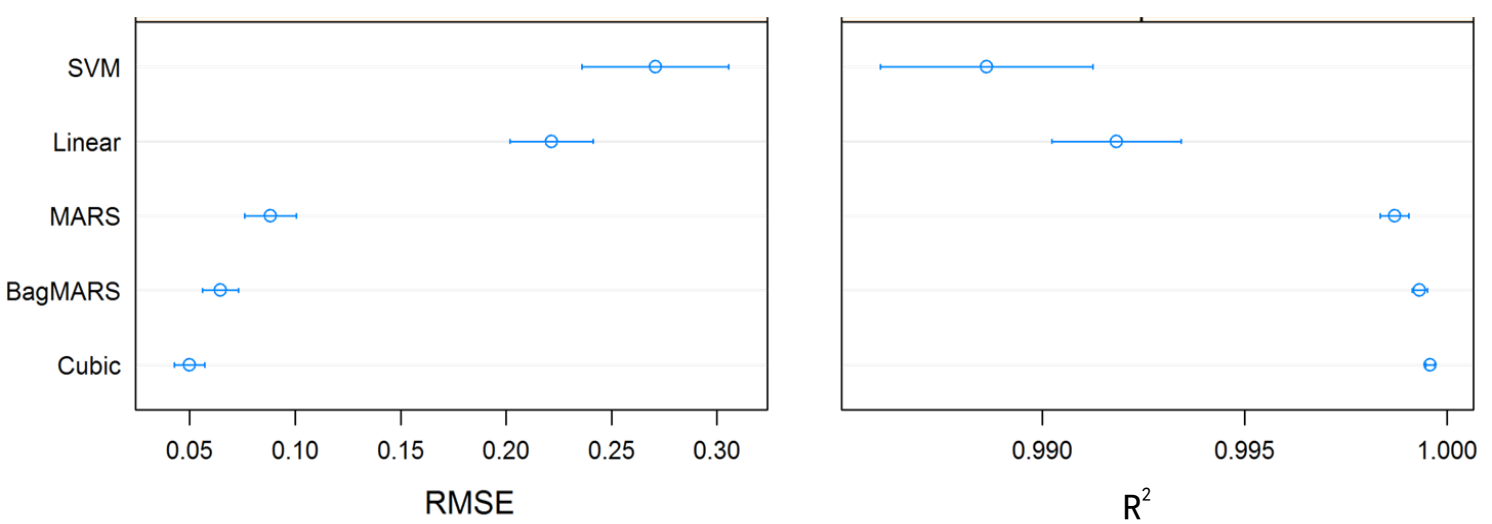

413

Confidence Level: $\mathbf{0 . 9 5}$

(b) Cooling energy use
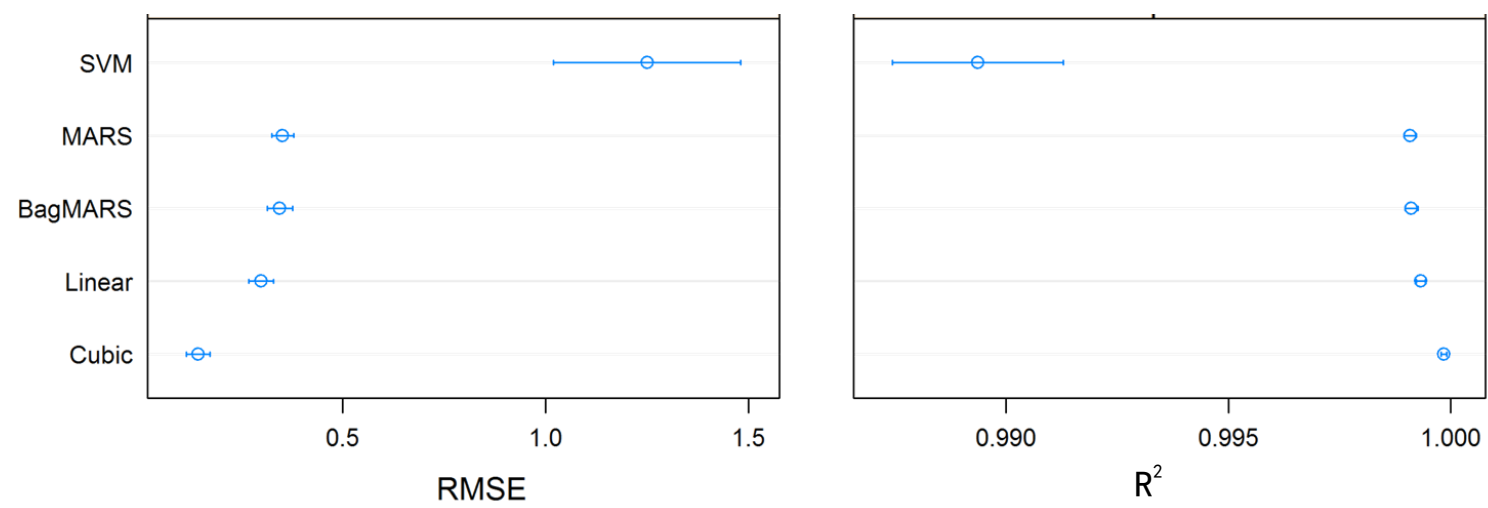

(c) Carbon emissions

Figure 3. Comparison of five machine learning models for estimating performance of building 

using external validation

\begin{tabular}{ccccccc}
\hline \multirow{2}{*}{ Model } & \multicolumn{2}{c}{ Heating energy } & \multicolumn{2}{c}{ Cooling energy } & \multicolumn{2}{c}{ Carbon emission } \\
\cline { 2 - 7 } & $\mathrm{R}^{2}$ & RMSE & $\mathrm{R}^{2}$ & RMSE & $\mathrm{R}^{2}$ & RMSE \\
\hline Linear & 0.992 & 0.326 & 0.991 & 0.223 & 0.999 & 0.300 \\
MARS & 0.998 & 0.176 & 0.998 & 0.085 & 0.999 & 0.343 \\
Bagging MARS & 0.998 & 0.162 & 0.999 & 0.065 & 0.999 & 0.343 \\
SVM & 0.990 & 0.365 & 0.990 & 0.245 & 0.987 & 1.315 \\
Cubist & 0.999 & 0.136 & 0.999 & 0.041 & 0.999 & 0.204 \\
\hline
\end{tabular}

Figure $3 \mathrm{~b}$ shows the comparison of five machine learning models for cooling energy use. The Cubist model is still the best performer in this case study, similar to heating energy use. The next model is the bagging MARS model, which is better than the MARS models in terms of both $\mathrm{R}^{2}$ and RMSE. Therefore, the bagging technique has more influence for cooling than heating energy in this office building by providing more stable predictions. The linear and SVM models do not perform as well as the other three learning models. The corresponding statistics of $\mathrm{R}^{2}$ and RMSE from external validation are summarized in Table 3 to indicate that the Cubist and MARS models are two best performers for predicting cooling energy in the office building. In terms of RMSE, the Cubist model is approximately 5 times better than the linear model.

Figure $3 \mathrm{c}$ shows the predictive performance of five machine learning models for carbon emissions using cross validation. The best learning model is from the Cubist method. The next model is from linear regression, which may be unexpected. This suggests the linear model may have better performance compared to non-parametric models when the relationship between inputs and outputs is approximately linear. The linear model shows slightly better performance than the MARS and bagging MARS models. As a result, the Cubist and linear models are selected to validate the model performance using external EnergyPlus simulation runs. The corresponding RMSE and $\mathrm{R}^{2}$ from external validation are listed in Table 3 to show that two best models are the Cubist and linear model in this case study to estimate carbon emissions.

The bootstrap approach is used as an alternative model selection method to compare the results from the cross-validation method. A bootstrap sample is a random sample of the original data set taken with replacement, which has the same size as the original data. Hence, the samples that are not selected in a bootstrap sample can be used as out-of-bag samples to validate models obtained from a bootstrap sample. The results indicate that most of results are similar to those obtained from the cross-validation method as shown in Figure 3 although the variations from the bootstrap approach are smaller than those from the cross-validation method.

It is recommended to determine the accuracy of machine learning models required for building projects in order to decide when to stop choosing suitable machine learning models. This is because a large number of machine learning models are available [33] and it is unnecessary to try a large number of machine learning models for a specific building project. The acceptance criterion for model performance can refer to the values set out by ASHRAE Guideline 14 in which the coefficient variations of RMSE for energy models should be lower than 5\% [47]. This threshold value has been widely used in building energy analysis [48]. The 
coefficient variations of RMSE for all three performance output (heating, cooling, and carbon emissions) are lower than this criterion in this case study. The mean coefficient variations of RMSE for Cubist models are 1.02\%, 0.13\%, and 0.09\% for heating, cooling, and carbon 461 emission, respectively. Therefore, the Cubist models have good performance in terms of ASHRAE Guideline 12 [47]. This criterion from ASHRAE can be regarded as the minimum requirement for performance of energy models. This is because buildings typically are bespoke, one-off products that are designed in response to a unique client brief. The translation of client brief to technical requirements is mostly conducted by expert consultants who have a considerable freedom in setting accuracy targets and thresholds [49], which is similar to the processes observed in Systems Engineering [50, 51].

\subsection{Results of uncertainty analysis}

\subsubsection{Annual heating energy}

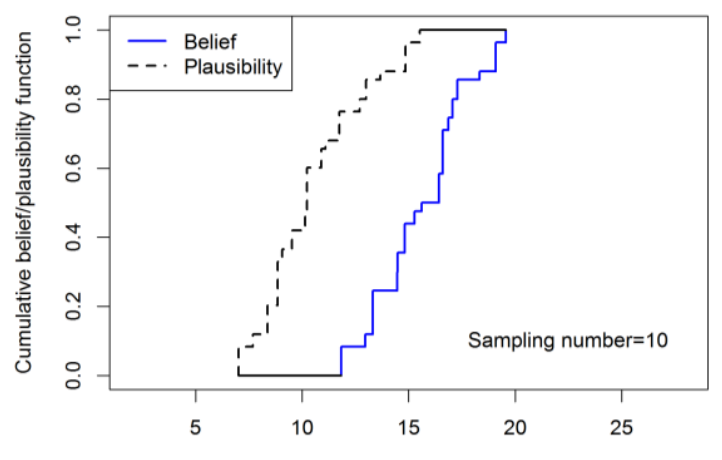

Heating energy use $(\mathrm{kWh} / \mathrm{m} 2)$

470

471

472

473

474 (e) sampling number 5,000
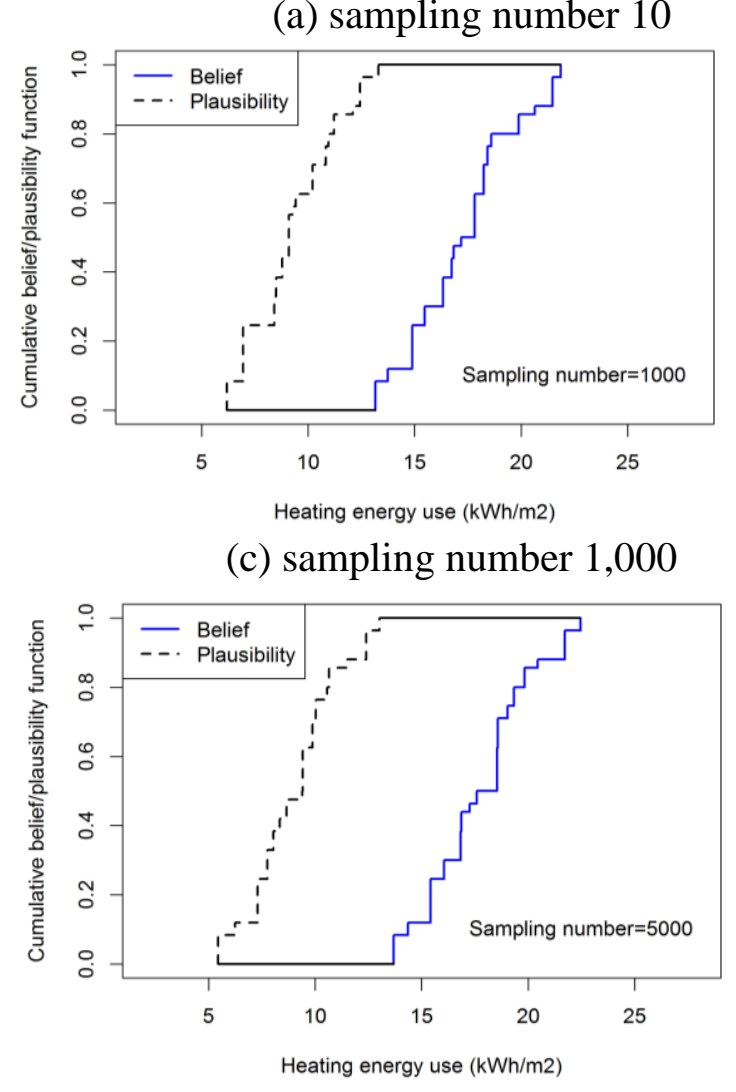

Heating energy use $(\mathrm{kWh} / \mathrm{m} 2)$

(c) sampling number 1,000

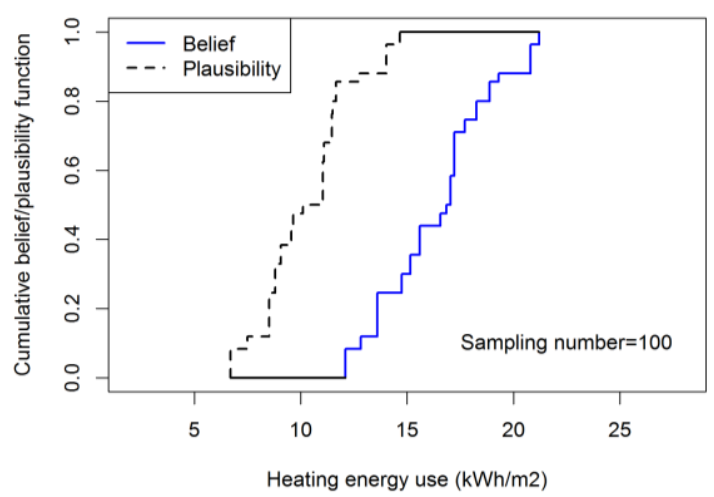

(b) sampling number 100

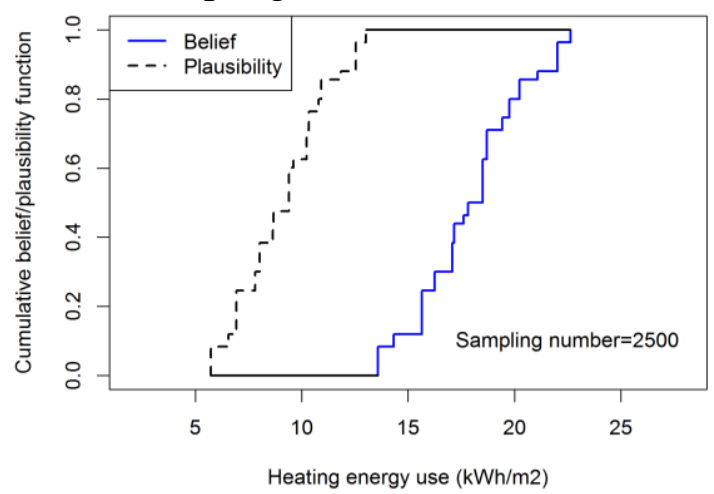

(d) sampling number 2,500

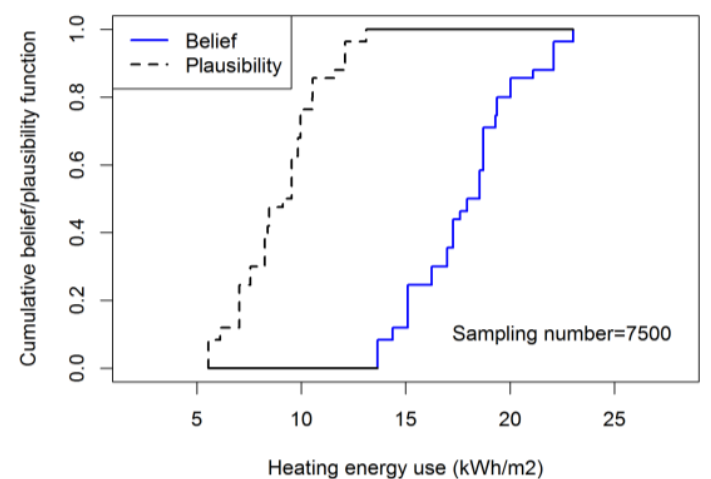

(f) sampling number 7,500 . 


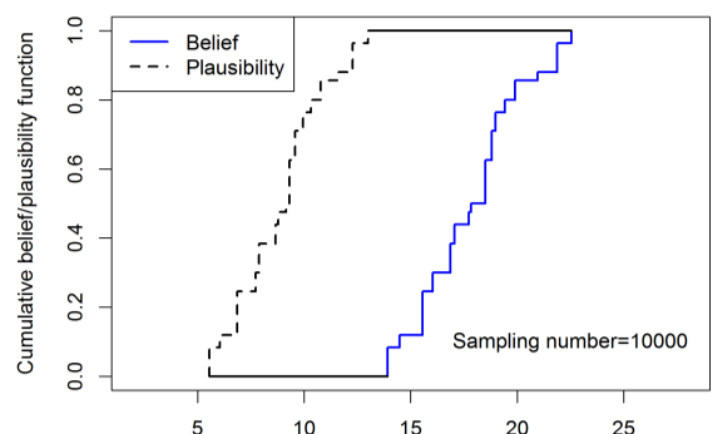

Heating energy use $(\mathrm{kWh} / \mathrm{m} 2)$

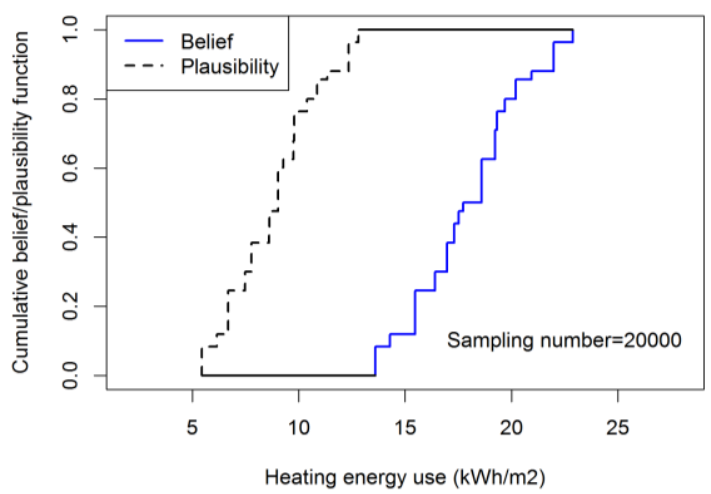

(h) sampling number 20,000

(g) sampling number 10,000

Figure 4. Comparison of stability of uncertainty results with sampling number for heating energy using the MARS model in case A

For uncertainty analysis, it is necessary to assess the convergence of results. Figure 4 compares change of cumulative belief function (CBF) and cumulative plausibility functions $(\mathrm{CPF})$ for annual heating energy using the MARS model as the sample size increases. The area between the CBF and the CPF becomes larger with an increase in sample size because more resamples are required to find the minimum and maximum values for every cell defined in the DST analysis. For the sampling size of 10, the results are apparently inadequate and not converged by comparing the shapes of CBF and CPF for the case of sampling size 10 and 100. As the sample size increases to 2,500, the uncertainty for the CBF and CPF tends to become stable. Beyond sample size of 5,000, the shapes of CBF and CPF only change slightly. To obtain fully converged results, sample size of 10,000 are used in this research for all three outputs: heating, cooling, and carbon emissions.

Figure 5 shows the uncertainty results for annual heating energy of the office building based on the Dempster-Shafer theory with a sample size 10,000. The shapes of cumulative belief function (CBF) and cumulative plausibility functions (CPF) from the Cubist and MARS models are similar for case A although the predicted values from the MARS model are slightly larger than those from the Cubist model. The same conclusion can be also obtained for the case B as illustrated in Figure 5c and Figure 5d. Hence, both the Cubist or MARS models can produce reliable results instead of using the computationally expensive EnergyPlus models.

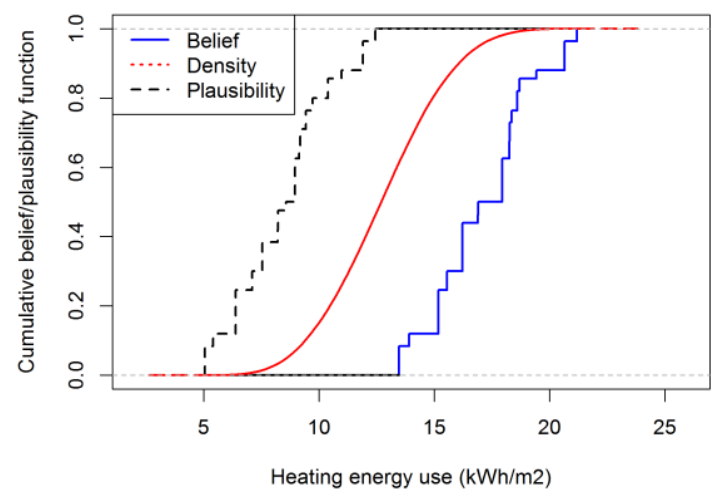

(a) Cubist model for case $\mathrm{A}$

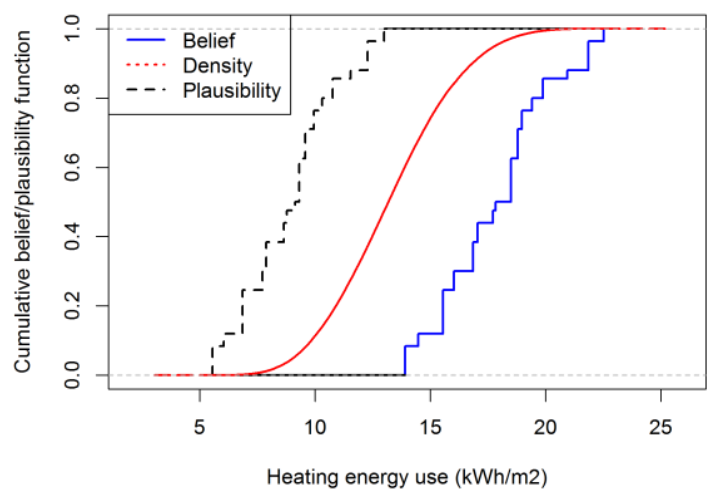

(b) MARS model for case A 


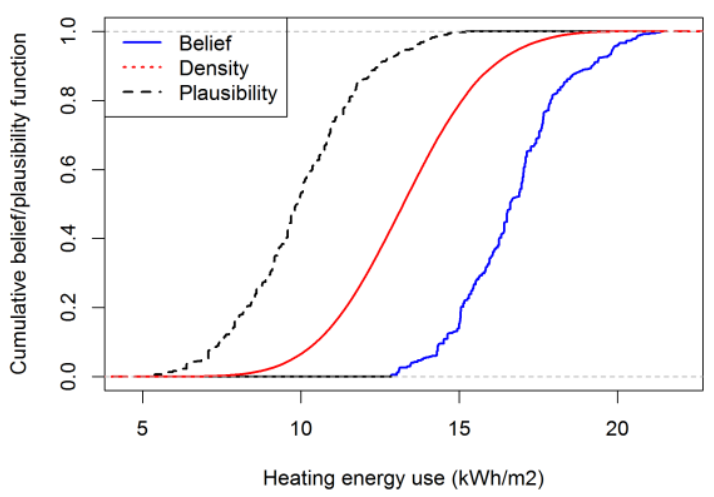

(c) Cubist model for case B

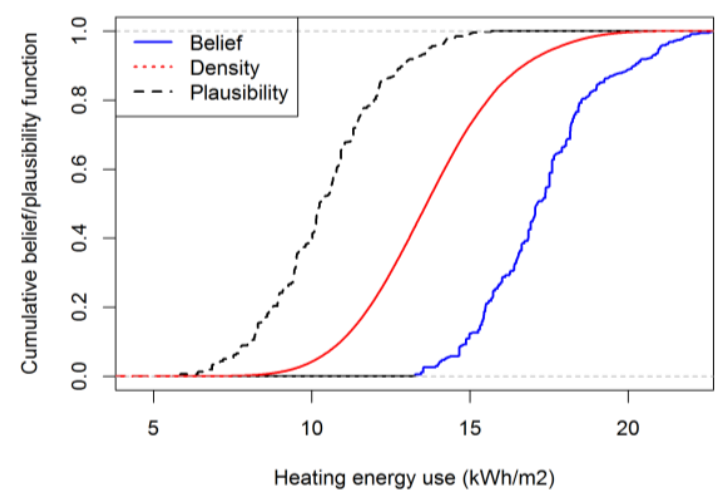

(d) MARS model for case B

Figure 5. Uncertainty analysis of annual heating energy from Dempster-Shafer theory

Based on the DST analysis, annual heating energy in this office building should be within the ranges of solid blue (CBF) and dashed black (CPF) lines in Figure 5. As might be expected, the red density plot (cumulative density function, $\mathrm{CDF}$ ) falls between the CBF and the CPF associated with the evidence space as defined in Table 1 and Table 2. This is because the CDF with uniform distribution (as described in section 3) is a special case of the DST results. For this office building, annual heating energy is unlikely to be more than $23 \mathrm{kWh} / \mathrm{m}^{2}$ and less than $5 \mathrm{kWh} / \mathrm{m}^{2}$. If the annual heating energy quota for office buildings (i.e. upper limit of energy use recommended or required by government) is $20 \mathrm{kWh} / \mathrm{m}^{2}$, then the lowest and highest probabilities above this quota in case A with the Cubist model are 0 and 12\%, respectively, as shown in Figure 5a. If using the MARS model, the corresponding lowest probability is the same as the Cubist model $(0 \%)$ and the highest probability is slightly higher, around $11 \%$.

As also can be seen from Figure 5, the CBF and CPF are more smooth for case B than for case A. This is in line with the input uncertainty defined in Table 2. A large number of hypercube for input variables in the DST approach means more discretization and smaller intervals, which usually results in smoother output. The number of hypercubes in case B is 512 , whereas the number of hypercube in case $\mathrm{A}$ is only 32 as described in section 2.1. As a result, a smoother CBF and CPF is observed for case B in Figure 5.

\subsubsection{Annual cooling energy}

Figure 6 shows the uncertainty results for cooling energy using the Cubist and bagging MARS models in case A and case B for the office building. The shapes of CBF and CPF are similar from the Cubist and bagging MARS models in case A. This statement also holds true in the case B. Hence, the uncertainty of output is reliable using machine learning models instead of engineering-based EnergyPlus models. 


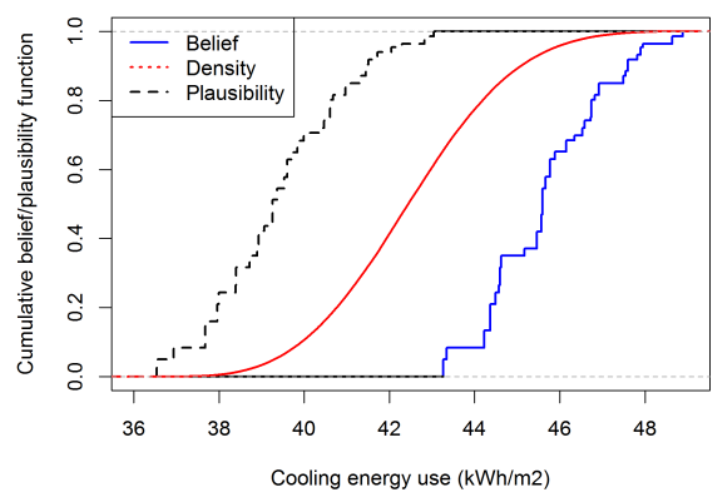

(a) Cubist model for case A

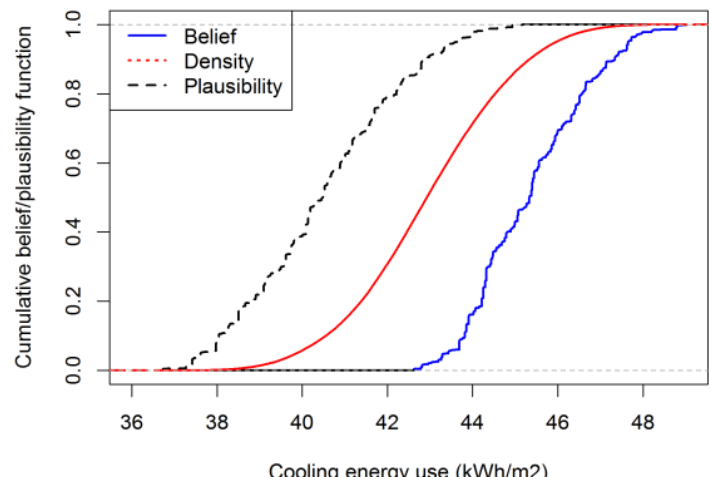

(c) Cubist model for case B number of inputs that leads to smoother outputs.

\subsubsection{Annual carbon emissions} emissions using the fast-computing learning models.

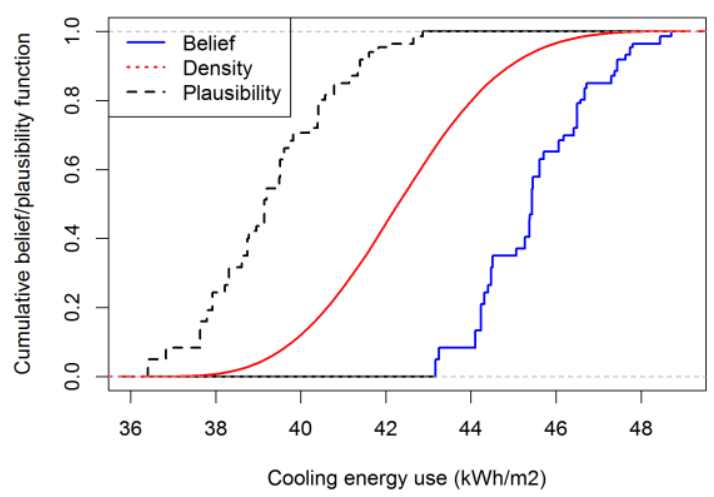

(b) Baging MARS model for case A

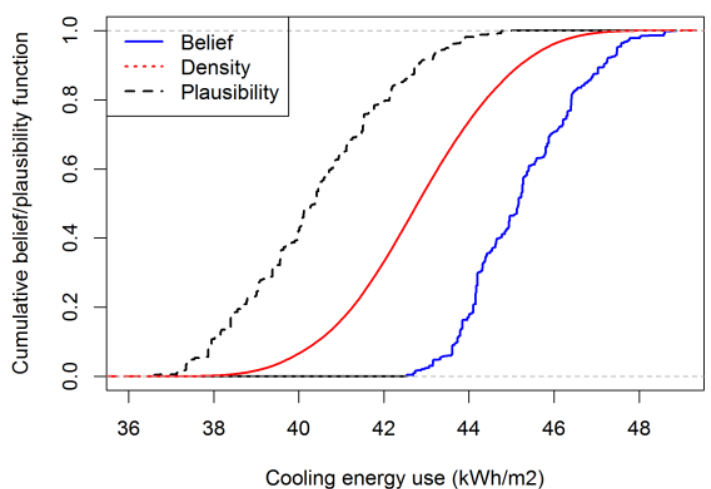

(d)Baging MARS model for case B

\section{Figure 6. Uncertainty analysis of annual cooling energy from Dempster-Shafer theory}

The CDF results lie between the CBF and CPF since the uniform distribution used for the CDF is one of possible choices in evidence space of input variables using the DST method. Cooling energy is between 36 and $49 \mathrm{kWh} / \mathrm{m}^{2}$ in this case study. If the quota of annual cooling energy is $46 \mathrm{kWh} / \mathrm{m}^{2}$ for the office building, then the highest probability for the cooling energy above this quota value is around 38\% using two machine learning models (Figure 6a and Figure 6b). Compared to the case A, there are less jumps for the CBF and CPF plots in the case B. As discussed in section 4.2.1, this is due to the increase of hypercube

Figure 7 shows uncertainty results of annual carbon emissions using the Cubist and linear models in case A and case B. The results from these two machine learning models are very close for both cases. Hence, the results are robust for showing the variation of carbon 


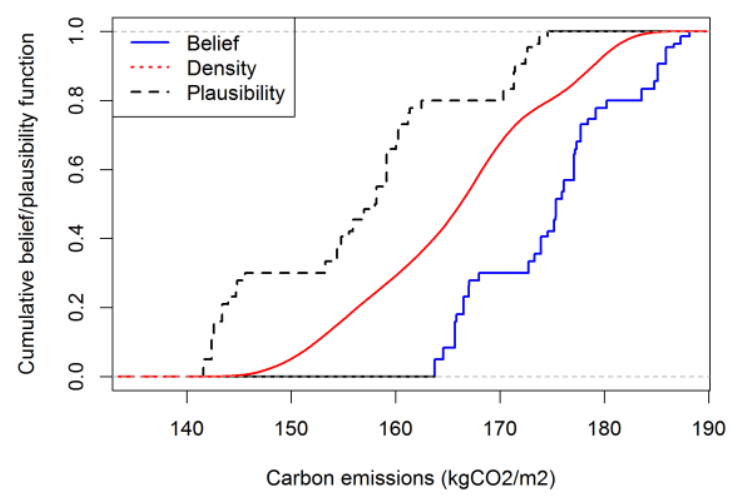

546 547

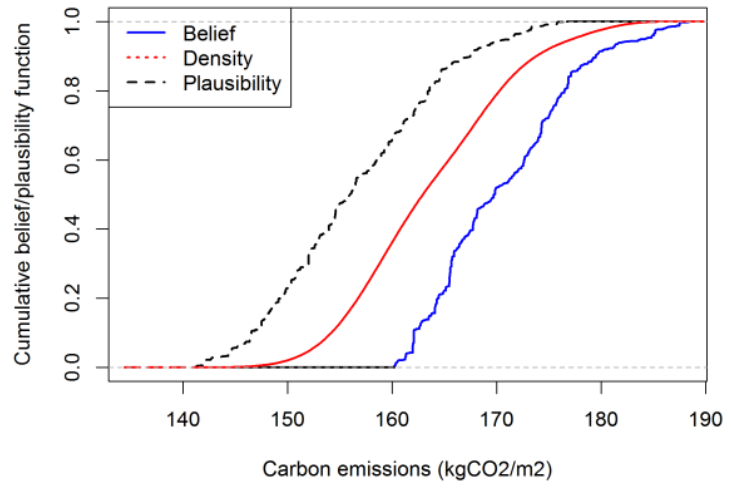

(c) Cubist model for case B in case $\mathrm{A}$. properly estimate uncertainty of building performance.

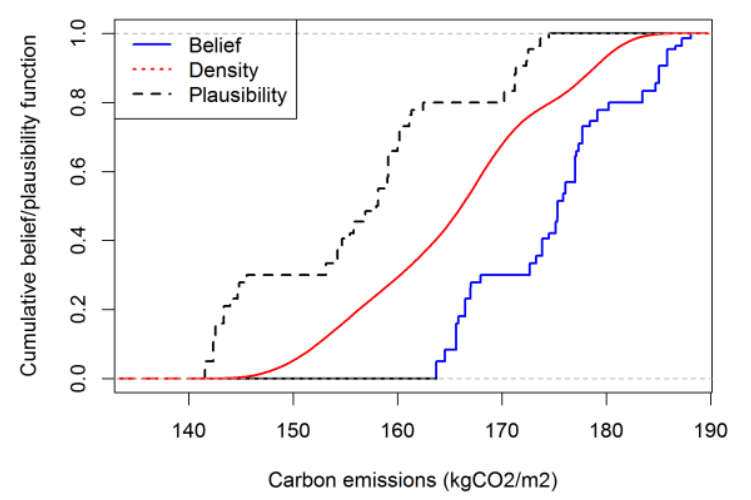

(a) Cubist model for case A

(b) Linear model for case A

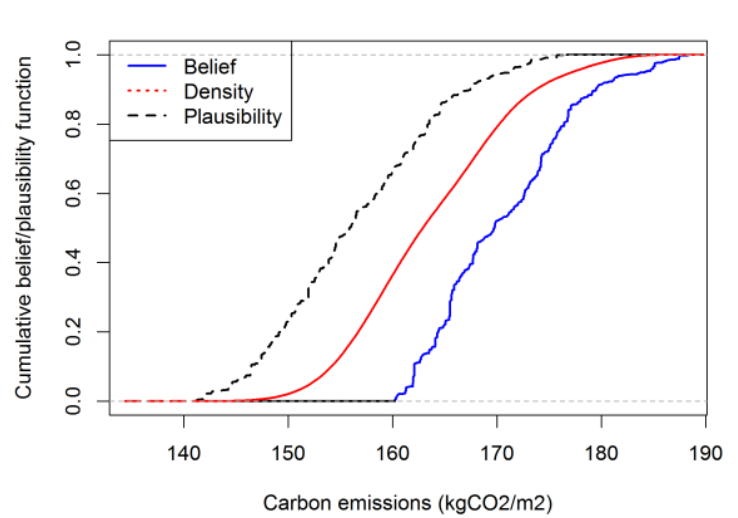

(d) Linear model for case B

\section{Figure 7. Uncertainty analysis of annual carbon emissions based on Dempster-Shafer theory}

It is apparent that in case A there are more obvious jumps in CBF and CPF for carbon emissions in comparison with heating and cooling energy use (Figure 5 and Figure 6). This can be explained by the sensitivity analysis as will be presented in section 4.3. The two dominant variables for carbon emissions are equipment and lighting heat gains that are listed in Table 1. As a result, the trends of carbon emissions are affected substantially by the specification of these two input variables. The equipment peak value has two discontinues intervals [13, 15] and [16, 17], which leads to significant jumps of CBF and CPF in Figure 7a and Figure $7 \mathrm{~b}$. The overlapping intervals from lighting peak values $([6,8]$ and $[7,9])$ also have important influence on carbon emissions. In case B (Table 2), the intervals for equipment and lighting equipment gains become more continuous by combining the opinions from two experts. Therefore, the $\mathrm{CBF}$ and $\mathrm{CPF}$ in case $\mathrm{B}$ become much smoother than those

Based on the analysis in this subsection, the results from DST analysis are different from the results of assuming uniform distributions for input variables in building energy assessment. The uncertainty from uniform distributions is significantly less than possible variations for building energy performance. Hence, when there is only limited information on input variables, the uniform distributions cannot be regarded as good choices for uncertainty analysis in building energy analysis. Instead, the DST analysis should be implemented to 


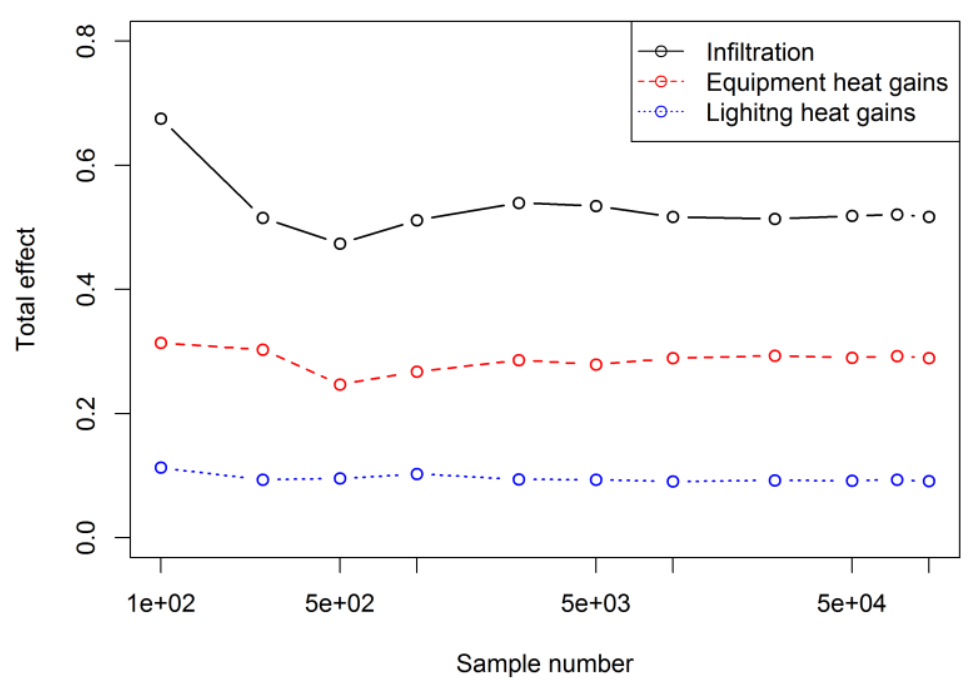

(a) Heating energy with the MARS model

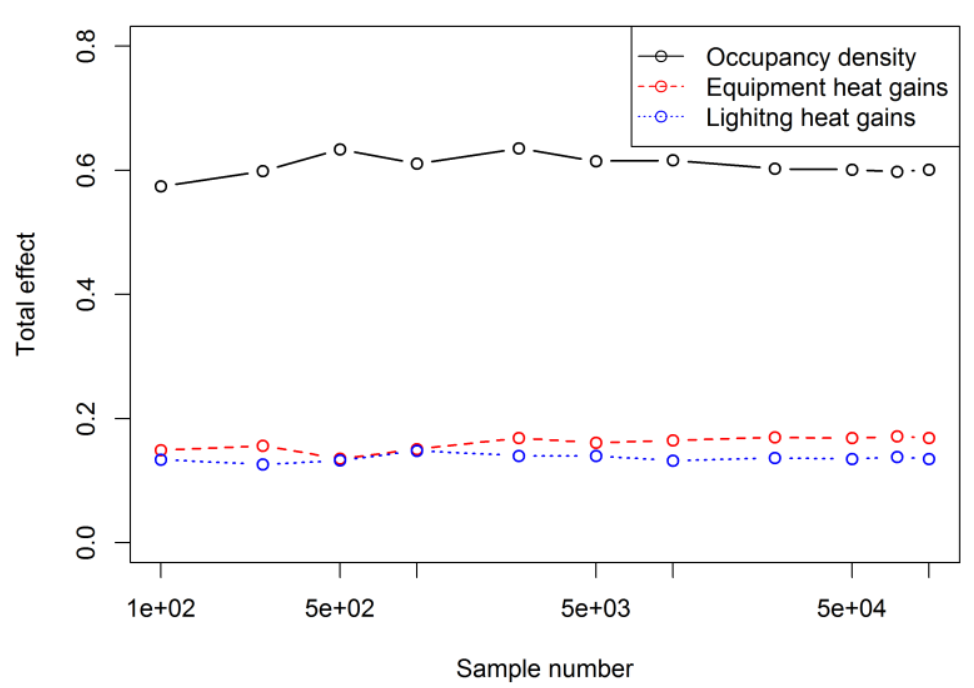

(b) Cooling energy with the Cubist model

Figure 8. Stability of total effects as a function of sample size from global sensitivity analysis

The two machine learning models with better predictive performance are used to provide robust results of sensitivity analysis for three performance measures: heating, cooling, and carbon emissions. The sensitivity analysis is implemented with a sample size of 100,000 from uncertain variables and the associated three distribution possibilities (uniform, left quadratic, and right quadratic) as discussed in section 2.3. Figure 8 demonstrates the stability of total effects from the Sobol global sensitivity analysis as a function of sample size. The results vary a lot at the sample sized below 2,500. In Figure 8b, there are intersections of ranking importance for equipment and lighting heat gains for cooling energy use at the sample size less than 1,000. After the sample size of 10,000, the total effects become stable in both Figure $8 \mathrm{a}$ and Figure $8 \mathrm{~b}$. The number of samples is chosen as 100,000 to confirm the convergence of sensitivity analysis. 


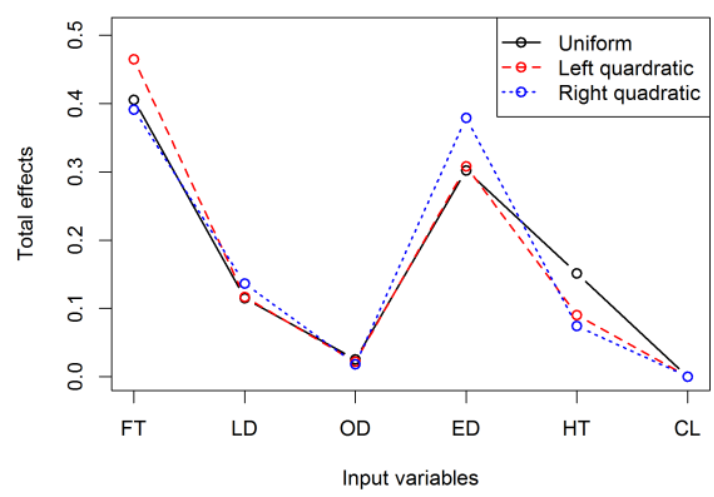

(a) Cubist model for case A

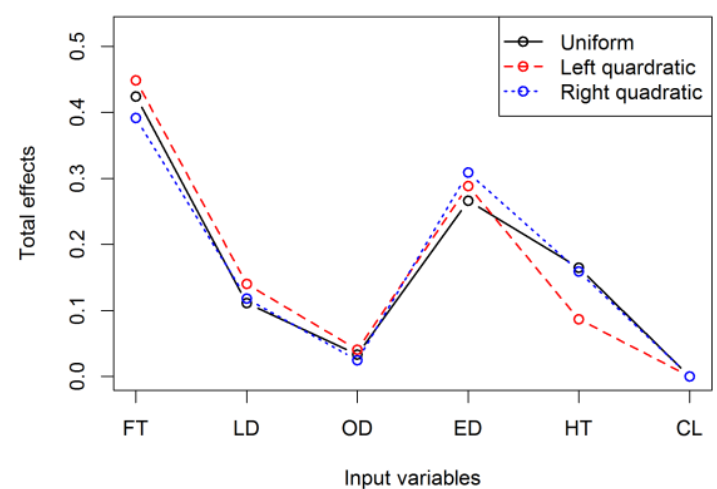

(c) Cubist model for case B

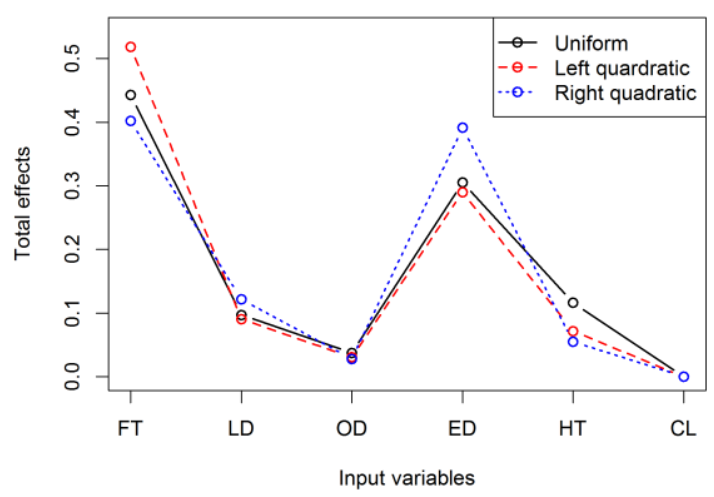

(b) MARS model for case A

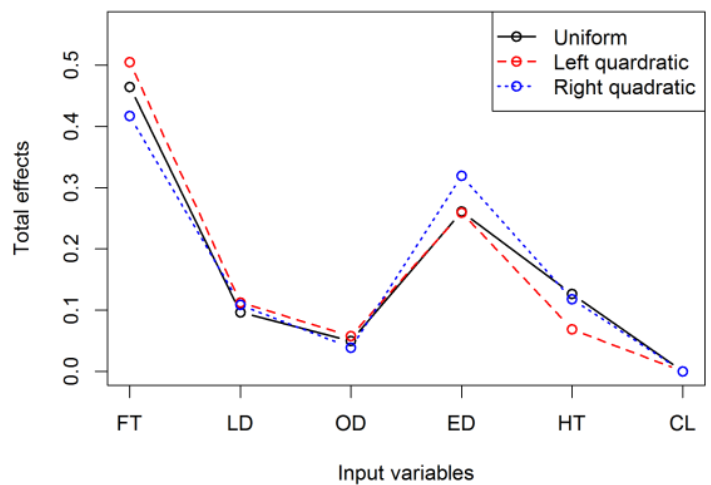

(d) MARS model for case B

\section{Figure 9. Results of sensitivity analysis for heating energy from Dempster-Shafer theory (refer to Table 1 for full names of input variables)}

Figure 9 shows the total effects of six variables with three types of distributions using the global Sobol sensitivity analysis for annual heating energy use in the office building. The ranking results are similar for three distribution possibilities as can be seen from Figure 9a. The ranking order is also similar from two machine learning models (Cubist and MARS) as illustrated in Figure 9a and Figure 9b. The most important variable identified here is the infiltration rate (FT), which accounts for approximate $40 \%$ of output variation. Hence, it is necessary to obtain reliable information on infiltration rate for accurately predicting annual heating energy. From the perspective of energy saving, it is important to try to reduce infiltration rate in order to reduce heating energy use. The next important variable is the equipment heat gains (ED) that also has important influences on annual heating energy use. For the right quadratic distributions, the importance from equipment heating gains becomes more evident and its important is in the same level as infiltration rate in the office building. The lighting heat gains (LD) and heating set-point temperatures (HT) have medium effects on heating energy use. The occupancy density (OD) and cooling heat-point (CL) have almost no influence on output variable in this case study. There are apparent similarities for ranking results in case A and case B. Hence, the ranking results of sensitivity analysis are not influenced by opinions from different experts although the results of uncertainty analysis are quite different in two cases as discussed in section 4.2.

Figure 10 shows the results of sensitivity analysis for annual cooling energy from two machine learning models in two cases. The Cubist and bagging MARS models present the similar results for ranking the importance of six variables influencing cooling energy use. The 
sensitivity rankings from case A are also similar to those from case B. In four subplots of Figure 10, the dominant variable is the occupancy density (OD), which accounts for around $60 \%$ of variations of annual cooling energy in the office building. Then, it is necessary to obtain the reliable data on occupancy density in order to provide accurate estimation on cooling energy use in this building. The next three variables have similar importance, equipment heat gains (ED), lighting heat gains (LD), and cooing set-point temperatures (CL). The remaining two variables (infiltration rate and heating set-point temperatures) have almost no effect on output variable. As also can be seen from Figure 10, the sensitivity results from three distributions are similar for cooling energy use in the office building. Hence, the assumption of various distributions does not influence the validity of ranking results in this case study.

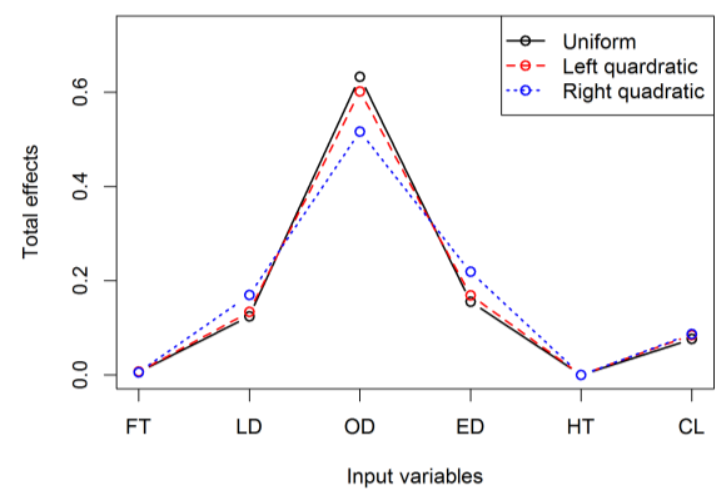

(a) Cubist model for case $\mathrm{A}$

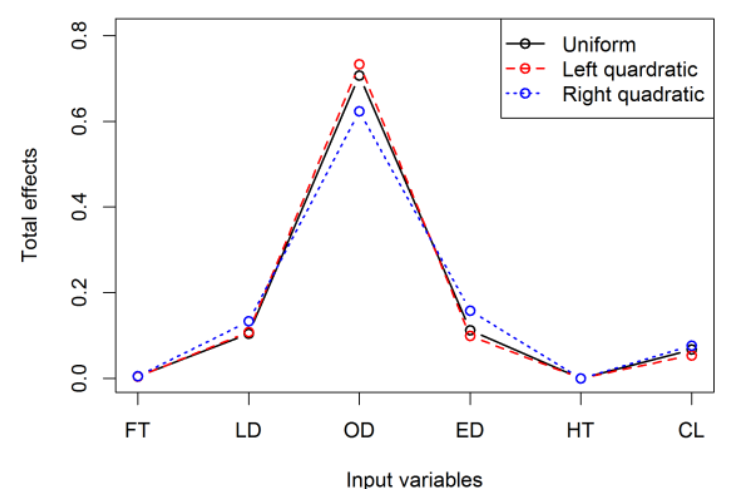

(c) Cubist model for case B

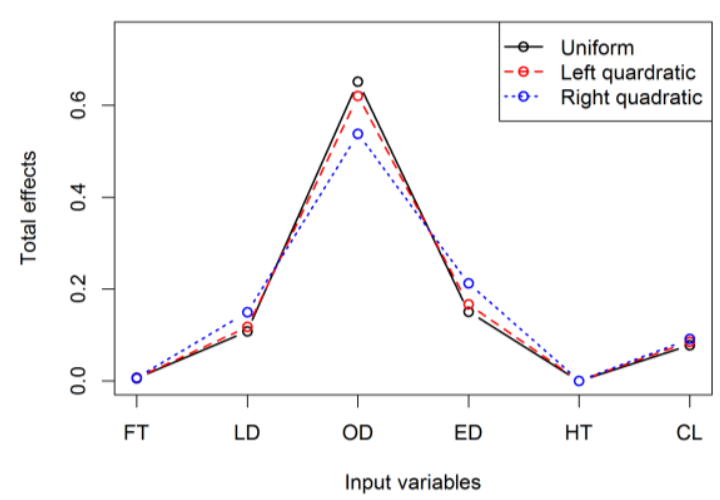

(b) Baging MARS model for case A

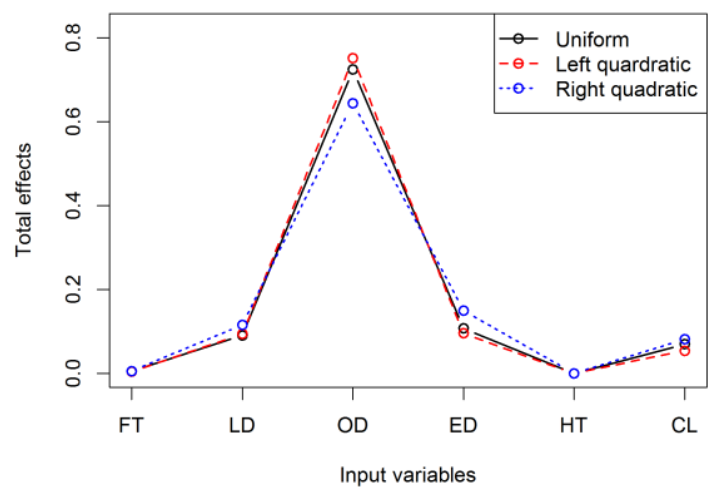

(d) Bagging MARS model for case B
Figure 10. Results of sensitivity analysis for cooling energy from Dempster-Shafer theory (refer to Table 1 for full names of input variables)

Figure 11 shows the ranking results from sensitivity analysis for annual carbon emissions from two machine learning models in two cases for this office building. As might be expected, equipment (ED) and lighting heat gains (LD) have significant influence on carbon emissions. This is because most of electricity use in office buildings is due to office equipment (such as computers, printing machine, projectors) and lighting. The variations of carbon emissions are almost not influenced by four remaining variables. It is also observed that the ranking results from the Cubist and linear models are very similar, which indicates that the sensitivity results obtained from this study are robust. In two cases, the trend of important variables is similar although three types of distributions lead to more disperse results in case $\mathrm{B}$ than those in case $\mathrm{A}$. 


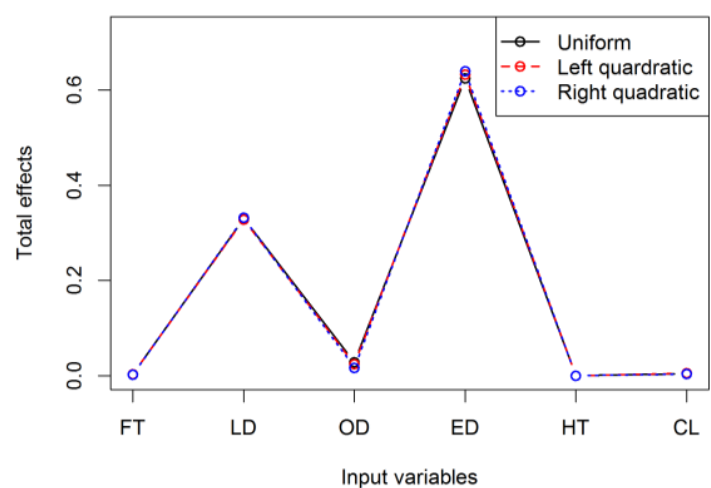

(a) Cubist model for case $\mathrm{A}$

643

644

645

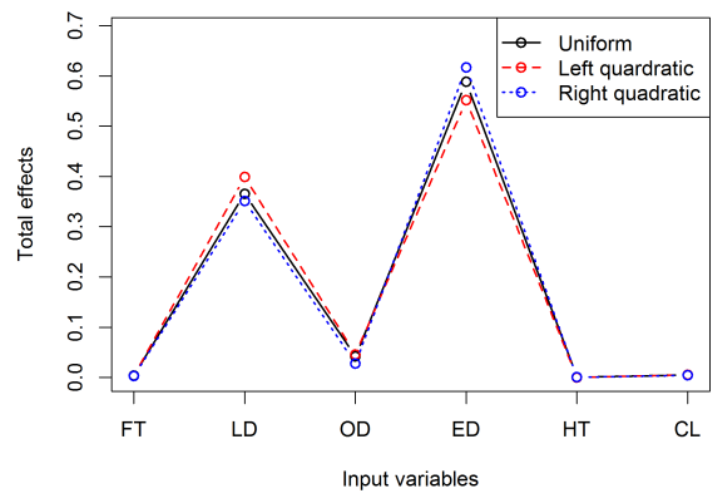

(c) Cubist model for case B method.

\section{CONCLUSIONS} building energy assessment.

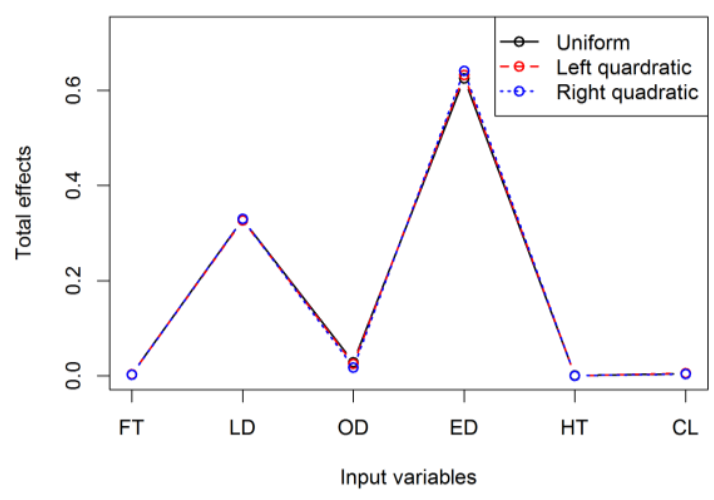

(b) Linear model for case A

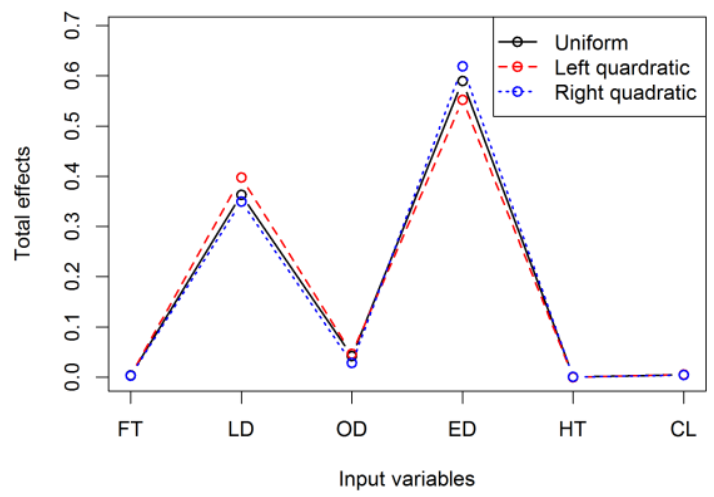

(d) Linear model for case B

Figure 11. Results of sensitivity analysis for carbon emissions from Dempster-Shafer theory (refer to Table 1 for full names of input variables)

Sensitivity analysis applied in this subsection would be useful to make informed decisions, depending on project purposes. For instance, energy saving measures can be determined even in the case of the availability of limited information by using DST sensitivity analysis since a spectrum of distributions for these input factors have been considered. If the aim of project is to reduce variations of energy performance, then more efforts should be made to collect more information on these key variables identified by the DST sensitivity

This research implements uncertainty and sensitivity analysis for assessing energy performance of an office building based on the DST (Dempster-Shafer theory) approach. Machine learning methods are used to expedite the computation since a large number of energy models needs to be run. The following conclusions can be drawn from this study.

(1) The DST analysis is applicable to provide informative uncertainty results of energy performance in buildings when only limited information on input variables is available. Note that the uncertainty results for energy use bounded between the CBF and CPF cannot be interpreted as being equally possible (similar to uniform distributions in probability theory). The energy performance may be any possible values within the intervals between the CBF and CPF based on the DST analysis. When the information on uncertainty of inputs is sufficient to specify distributions, the Monte-Carlo based sampling method is preferred in 
(2) Machine learning algorithms can be used to reduce high computational cost in implementing DST analysis in building energy analysis, instead of directly using engineeringbased energy models. It is recommended to compare several machine leaning methods and then choose at least two learning methods that are inherently different in nature in order to provide robust analysis.

(3) The DST analysis does not assume any distribution within the intervals of input factors. Hence, a spectrum of distributions should be used in implementing sampling-based sensitivity analysis to provide reliable sensitivity results for building energy analysis.

(4) It is necessary to assess the stability of results as a function of sample size from uncertainty and sensitivity analysis in applying the DST into building energy assessment. This is often ignored in applying uncertainty and sensitivity analysis in the field of building energy analysis.

The conclusions obtained above and the methods proposed in this paper can be applied to other buildings in various climate zones. However, there are several issues that still need to be addressed in further studies. One is to compare the performance of more machine learning algorithms (such as deep learning and ensemble learning methods) when applying them in DST analysis of building energy performance. Another issue is to investigate whether it is possible to determine the rule-of-thumb sample size (for instance, in terms of variable number) on applying the DST method in building energy analysis in order to make this method more readily available.

\section{ACKNOWLEDGEMENT}

This research was supported by the National Natural Science Foundation of China (No. 51778416) and the Key Projects of Philosophy and Social Sciences Research, Ministry of Education (China) "Research on Green Design in Sustainable Development" (contract No. 16JZDH014, approval No. 16JZD014).

\section{REFERENCES}

[1] S. Kiluk. Diagnostic information system dynamics in the evaluation of machine learning algorithms for the supervision of energy efficiency of districtheating-supplied buildings. Energy Conversion and Management. 150 (2017) 904-13.

[2] M. Heidarinejad, J.G. Cedeño-Laurent, J.R. Wentz, N.M. Rekstad, J.D. Spengler, J. Srebric. Actual building energy use patterns and their implications for predictive modeling. Energy Conversion and Management. 144 (2017) 164-80.

[3] W. Tian, X. Meng, B. Yin, Y. Sun, Y. Liu, X. Fu. Design of Robust Green Buildings Using a Non-probabilistic Uncertainty Analysis Method. Procedia Engineering. 205 (2017) 1049-55.

[4] F.R. Cecconi, M. Manfren, L.C. Tagliabue, A.L.C. Ciribini, E. De Angelis. Probabilistic behavioral modeling in building performance simulation: A Monte Carlo approach. Energy and Buildings. 148 (2017) 128-41.

[5] R. Choudhary, W. Tian. Influence of district features on energy consumption in nondomestic buildings. Building Research \& Information. 42 (2014) 32-46.

[6] J. Chen, G. Augenbroe, X. Song. Evaluating the potential of hybrid ventilation for small to medium sized office buildings with different intelligent controls and uncertainties in US climates. Energy and Buildings. 158 (2018) 1648-61.

[7] W. Tian, X. Fu, Y. Sun, B. Yin, X. Meng, Y. Liu. Sustainable Building Design Based on the Second Order Probability Approach. Procedia Engineering. 205 (2017) 1056-63.

[8] Y. Heo, G. Augenbroe, D. Graziano, R.T. Muehleisen, L. Guzowski. Scalable methodology for large scale building energy improvement: Relevance of calibration in modelbased retrofit analysis. Building and Environment. 87 (2015) 342-50. 
[9] L. Urbanucci, D. Testi. Optimal integrated sizing and operation of a CHP system with and Management. 157 (2018) 307-16.

[10] W. Tian, S. Yang, J. Zuo, Z. Li, Y. Liu. Relationship between built form and energy performance of office buildings in a severe cold Chinese region. Building simulation. 10 (2017) 11-24.

[11] G.A. Faggianelli, L. Mora, R. Merheb. Uncertainty quantification for Energy Savings Performance Contracting: Application to an office building. Energy and Buildings. 152 (2017) 61-72.

[12] C.J. Hopfe, J.L.M. Hensen. Uncertainty analysis in building performance simulation for design support. Energy and Buildings. 43 (2011) 2798-805.

[13] W. Tian, Y. Heo, P. de Wilde, Z. Li, D. Yan, C.S. Park, et al. A review of uncertainty analysis in building energy assessment. Renewable and Sustainable Energy Reviews. 93 (2018) 285-301.

[14] J.C. Helton, J.D. Johnson, W.L. Oberkampf, C.B. Storlie. A sampling-based computational strategy for the representation of epistemic uncertainty in model predictions with evidence theory. Computer Methods in Applied Mechanics and Engineering. 196 (2007) 3980-98.

[15] C. Xie, G. Li, F. Wei. An integrated QMU approach to structural reliability assessment based on evidence theory and kriging model with adaptive sampling. Reliability Engineering \& System Safety. 171 (2018) 112-22.

[16] P. Masoudi, T. Aïfa, H. Memarian, B. Tokhmechi. Uncertainty assessment of volumes of investigation to enhance the vertical resolution of well-logs. Journal of Petroleum Science and Engineering. 154 (2017) 252-76.

[17] X. Zhao, K. Mu, F. Hui, C. Prehofer. A cooperative vehicle-infrastructure based urban driving environment perception method using a D-S theory-based credibility map. Optik International Journal for Light and Electron Optics. 138 (2017) 407-15.

[18] T.-J. Park, J.-H. Chang. Dempster-Shafer theory for enhanced statistical model-based voice activity detection. Computer Speech \& Language. 47 (2018) 47-58.

[19] J. Chaney, E. Hugh Owens, A.D. Peacock. An evidence based approach to determining residential occupancy and its role in demand response management. Energy and Buildings. 125 (2016) 254-66.

[20] Y. Kim, K. Ahn, C. Park. Aggregated epistemic uncertainty for building energy prediction using Dempster-Shafer evidence theory. Proceedings of 9th IAQVEC (Indoor Air Quality Ventilation \& Energy Conservation In Buildings) Conference October 23-26, 2016, Incheon, Korea. , 2016.

[21] DOE. EnergyPlus V8.8, September 2017, Department of Energy, USA. 2017.

[22] Y. Kwak, J.-H. Huh. Development of a method of real-time building energy simulation for efficient predictive control. Energy Conversion and Management. 113 (2016) 220-9. [23] W. Oberkampf, J.C. Helton. Chapter 10: Evidence Theory for Engineering Applications. in: E. Nikolaidis, D.M. Ghiocel, S. Singhal, (Eds.), Engineering Design Reliability Handbook. CRC Press2004. pp. 269-85.

[24] H.-R. Bae, R.V. Grandhi, R.A. Canfield. Epistemic uncertainty quantification techniques including evidence theory for large-scale structures. Computers \& Structures. 82 (2004) 110112.

[25] W. Tian, P.D. Wilde. Impact of global warming on thermal performance of domestic buildings using probabilistic climate data. International Journal of Global Warming. 10 (2016) 514-35.

[26] J.C. Helton, J.D. Johnson, C. Sallaberry, C.B. Storlie. Survey of sampling-based methods for uncertainty and sensitivity analysis. Reliability Engineering \& System Safety. 91 (2006) 1175-209. 
[27] W. Tian, J. Song, Z. Li, P. de Wilde. Bootstrap techniques for sensitivity analysis and model selection in building thermal performance analysis. Applied Energy. 135 (2014) 320-8. [28] S. Heidenreich, H. Gross, M. Bär, L. Wright. Uncertainty propagation in computationally expensive models: A survey of sampling methods and application to scatterometry. Measurement. 97 (2017) 79-87. [29] M. Kuhn. Building predictive models in R using the caret package. Journal of Statistical Software. 28 (2008) 1-26. [30] L. Wei, W. Tian, E.A. Silva, R. Choudhary, Q. Meng, S. Yang. Comparative Study on Machine Learning for Urban Building Energy Analysis. Procedia Engineering. 121 (2015) 285-92. probabilistic climate projections: A UK case study. Automation in Construction. 20 (2011) 1096-109.

[32] W. Tian, S. Yang, Z. Li, S. Wei, W. Pan, Y. Liu. Identifying informative energy data in Bayesian calibration of building energy models. Energy and Buildings. 119 (2016) 363-76.

[33] M. Kuhn, K. Johnson. Applied predictive modeling. Springer2013.

[34] T. Hastie, R. Tibshirani, J. Friedman. The Elements of Statistical Learning: Data Mining, Inference, and Prediction. Springer2009.

[35] J. Song, L. Wei, Y. Sun, W. Tian. Implementation of Meta-modelling for Sensitivity Analysis in Building Energy Analysis. The eSim 2014 conference, , May 7 to 10, Ottawa, Canada, 2014.

[36] W. Tian, R. Choudhary, G. Augenbroe, S.H. Lee. Importance analysis and meta-model construction with correlated variables in evaluation of thermal performance of campus buildings. Building and Environment. 92 (2015) 61-74.

[37] A. Saltelli, M. Ratto, T. Andres, F. Campolongo, J. Cariboni, D. Gatelli, et al. Global sensitivity analysis: the primer. Wiley-Interscience 2008.

[38] J.C. Helton, J.D. Johnson, W.L. Oberkampf, C.J. Sallaberry. Sensitivity analysis in conjunction with evidence theory representations of epistemic uncertainty. Reliability Engineering \& System Safety. 91 (2006) 1414-34.

[39] A. Saltelli. Making best use of model evaluations to compute sensitivity indices. Computer Physics Communications. 145 (2002) 280-97.

[40] B.I. Gilles Pujol, Alexandre Janon. R package sensitivity V1.12.2: sensitivity: Global Sensitivity Analysis of Model Outputs. https://CRAN.R-project.org/package=sensitivity accessed on 2016-11-20. 2016.

[41] MOC. GB50189-2015. Design standard for energy efficiency of public buildings, Ministry of Construction (MOC) of P.R.China China Planning Press (2015) (in Chinese)2015. [42] MOC. GB50189-2005. Energy Conservation Design Regulation for Public Buildings. Ministry of Construction (MOC) of P.R.China China Planning Press (2005) (in Chinese)2005. [43] NDRC. China regional grid based line emission factor in 2016 (in Chinese), by NDRC (National Development and Reform Comission). 2017.

[44] S. Yang, W. Tian, L. Wei, Z. Wang. Uncertainty analysis of carbon emissions in food industry. Food Research and Development. 37 (2016) 215-8, in Chinese.

[45] K. Sentz, S. Ferson. Combination of evidence in Dempster-Shafer Theory, Report No. SAND2002-0835, Sandia National Laboratories. 2002.

[46] W. Jiang, J. Zhan. A modified combination rule in generalized evidence theory. Applied Intelligence. 46 (2017) 630-40.

[47] ASHRAE. Guideline 14-2002 Measurement of Energy and Demand Savings. ASHRAE, Atlanta, GA. (2002).

[48] D. Coakley, P. Raftery, M. Keane. A review of methods to match building energy simulation models to measured data. Renewable and Sustainable Energy Reviews. 37 (2014) 123-41. 
822 [49] P. de Wilde. Building performance analysis. . Wiley-Blackwell. 2018.

823 [50] INCOSE. INCOSE (International Council on Systems Engineering) Systems 824 Engineering Handbook: A Guide for System Life Cycle Processes and Activities. . Wiley. 8252015.

826 [51] T. Gilb. Competitive engineering: a handbook for systems engineering, requirements engineering, and software engineering using planguage. . Butterworth-Heinemann. 2005. 